\begin{tabular}{|c|l|}
\hline Title & Ground-state properties of neutron-rich Mg isotopes \\
\hline Author(s) & $\begin{array}{l}\text { Watanabe, S.; Minomo, K.; Shimada, M.; Tagami, S.; Kimura, M.; Takechi, M.; Fukuda, M.; Nishimura, D.; Suzuki, } \\
\text { T.; Matsumoto, T.; Shimizu, Y. R.; Y ahiro, M. }\end{array}$ \\
\hline Citation & $\begin{array}{l}\text { Physical Review C, 89(4), O44610 } \\
\text { https://doi.org/L0.1103/PhysRevC.89.044610 }\end{array}$ \\
\hline Issue Date & 201404_23 \\
\hline Doc URL & http://hdl.handle.net/2115/56533 \\
\hline Rights & @2014American Physical Society \\
\hline Type & article \\
\hline File Information & PhysRevC_89_044610.pdf \\
\hline
\end{tabular}

Instructions for use 


\title{
Ground-state properties of neutron-rich Mg isotopes
}

\author{
S. Watanabe,${ }^{1,{ }^{*}}$ K. Minomo, ${ }^{1}$ M. Shimada,${ }^{1}$ S. Tagami, ${ }^{1}$ M. Kimura,${ }^{2}$ M. Takechi,${ }^{3,4}$ M. Fukuda,${ }^{5}$ D. Nishimura, ${ }^{6}$ T. Suzuki, \\ T. Matsumoto, ${ }^{1}$ Y. R. Shimizu, ${ }^{1}$ and M. Yahiro ${ }^{1}$ \\ ${ }^{1}$ Department of Physics, Kyushu University, Fukuoka 812-8581, Japan \\ ${ }^{2}$ Department of Physics, Hokkaido University, Sapporo 060-0810, Japan \\ ${ }^{3}$ Gesellschaft für Schwerionenforschung GSI, 64291 Darmstadt, Germany \\ ${ }^{4}$ RIKEN, Nishina Center, Wako, Saitama 351-0106, Japan \\ ${ }^{5}$ Department of Physics, Osaka University, Osaka 560-0043, Japan \\ ${ }^{6}$ Department of Physics, Tokyo University of Science, Chiba 278-8510, Japan \\ ${ }^{7}$ Department of Physics, Saitama University, Saitama 338-8570, Japan
}

(Received 10 March 2014; published 23 April 2014)

\begin{abstract}
We analyze recently measured total reaction cross sections for ${ }^{24-38} \mathrm{Mg}$ isotopes incident on ${ }^{12} \mathrm{C}$ targets at $240 \mathrm{MeV} /$ nucleon by using the folding model and antisymmetrized molecular dynamics (AMD). The folding model well reproduces the measured reaction cross sections, when the projectile densities are evaluated by the deformed Woods-Saxon (def-WS) model with AMD deformation. Matter radii of ${ }^{24-38} \mathrm{Mg}$ are then deduced from the measured reaction cross sections by fine tuning the parameters of the def-WS model. The deduced matter radii are largely enhanced by nuclear deformation. Fully microscopic AMD calculations with no free parameter well reproduce the deduced matter radii for ${ }^{24-36} \mathrm{Mg}$, but still considerably underestimate them for ${ }^{37,38} \mathrm{Mg}$. The large matter radii suggest that ${ }^{37,38} \mathrm{Mg}$ are candidates for deformed halo nucleus. AMD also reproduces other existing measured ground-state properties (spin parity, total binding energy, and one-neutron separation energy) of $\mathrm{Mg}$ isotopes. Neutron-number $(N)$ dependence of deformation parameter is predicted by AMD. Large deformation is seen from ${ }^{31} \mathrm{Mg}$ with $N=19$ to a drip-line nucleus ${ }^{40} \mathrm{Mg}$ with $N=28$, indicating that both the $N=20$ and 28 magicities disappear. $N$ dependence of neutron skin thickness is also predicted by AMD.
\end{abstract}

DOI: 10.1103/PhysRevC.89.044610

PACS number(s): 21.10.Gv, 21.60.Ev, 21.60.Gx, 25.60.-t

\section{INTRODUCTION}

Elucidation of properties of unstable nuclei is an important subject in nuclear physics. Some exotic properties were found so far in unstable nuclei particularly near the drip line. One is the change of well-known magicity. The standard shell ordering can evolve as a function of neutron number $N$ or proton number $Z$ due to residual nucleon-nucleon interactions. This leads to a quenching of some shell gaps and consequently the change of magic numbers. When the abrupt onset of the change of magic numbers appears in a region, the region is called the island of inversion. In the region, nuclei have larger binding energies than expected due to collectivity such as rotation.

As a pioneering work, Klapisch and Thibault first revealed anomalies in the binding energies of neutron-rich $\mathrm{Na}$ isotopes [1,2], and Warburton et al. predicted that $(s d)^{-2}(f p)^{2}$ intruder configurations form the ground states in the $N=20 \sim 22$ region of $\mathrm{Ne}, \mathrm{Na}$, and $\mathrm{Mg}$ isotopes [3]. This prediction was supported by mass measurements [4]. In the region, strong deformation of nuclei was suggested by measured low excitation energies and large $B(E 2)$ values of the first excited states [5-9]. As a mechanism behind the shell-gap quenching, more recently, the importance of the nucleon-nucleon tensor interaction was pointed out by Otsuka et al. [10,11]. The $N=20$ magicity is thus considered to disappear in $\mathrm{Ne}, \mathrm{Na}$, and $\mathrm{Mg}$ isotopes.

\footnotetext{
*s-watanabe@phys.kyushu-u.ac.jp
}

Another important progress in the physics of unstable nuclei is the discovery of halo nuclei by measurements of interaction cross sections $\sigma_{\mathrm{I}}$ or one- and two-neutron removal cross sections [12-14]. Here $\sigma_{\mathrm{I}}$ is used as a substitute of reaction cross sections $\sigma_{\mathrm{R}}$, since the two cross sections are nearly identical for the scattering of unstable nuclei at intermediate and high incident energies [15]. Now, ${ }^{6} \mathrm{He},{ }^{11} \mathrm{Li}$, ${ }^{11} \mathrm{Be}$ and others are considered to be halo nuclei. Recently, Nakamura et al. [16] suggested through measurements of the one-neutron removal cross section of ${ }^{31} \mathrm{Ne}$ on $\mathrm{C}$ and $\mathrm{Pb}$ targets at $240 \mathrm{MeV} /$ nucleon that ${ }^{31} \mathrm{Ne}$ is a halo nucleus that resides in the island of inversion. Takechi et al. [17] measured $\sigma_{\mathrm{I}}$ for $\mathrm{Ne}$ isotopes incident on ${ }^{12} \mathrm{C}$ targets at $240 \mathrm{MeV} /$ nucleon and came to the same conclusion as Nakamura et al. Very recently, Takechi et al. measured $\sigma_{\mathrm{R}}$ for ${ }^{24-38} \mathrm{Mg}$ isotopes on $\mathrm{C}$ targets at $240 \mathrm{MeV} /$ nucleon [18] and suggested that ${ }^{37} \mathrm{Mg}$ is a halo nucleus.

A powerful tool of analyzing measured $\sigma_{\mathrm{R}}$ or $\sigma_{\mathrm{I}}$ microscopically is the folding model with the $g$-matrix effective nucleon-nucleon interaction [19-29]. For nucleon scattering from stable target nuclei, the folding potential with the Melbourne $g$-matrix interaction reproduces measured elastic and reaction cross sections systematically with no adjustable parameter [26,29]. The folding model is reliable also for the scattering of unstable nuclei from stable target nuclei at intermediate incident energies, say $200 \mathrm{MeV} /$ nucleon, since the projectile breakup is small there. In fact, for ${ }^{31} \mathrm{Ne}$ scattering from ${ }^{12} \mathrm{C}$ targets at $240 \mathrm{MeV} /$ nucleon, the breakup cross section is about $1 \%$ of $\sigma_{\mathrm{R}}$ [30]. This indicates that the folding model is reliable also for other nucleus-nucleus scattering at 
intermediate energies, since ${ }^{31} \mathrm{Ne}$ is one of the most weakly bound systems.

The optical potential of nucleus-nucleus scattering is obtained by folding the $g$ matrix with the projectile and target densities. When the projectile is deformed, the density profile is elongated by the deformation. The elongation enlarges the root-mean-square (rms) radius of the projectile and eventually $\sigma_{\mathrm{R}}$. Recently, antisymmetrized molecular dynamics (AMD) with the Gogny-D1S interaction [31] was applied to nuclei in the island of inversion [32,33]. The calculations with the angular momentum projection (AMP) show that the nuclei are largely deformed. The result is consistent with that of the Hartree-Fock-Bogoliubov calculations with the AMP [34,35]. Here $n$-particle $m$-hole excitations in the Nilsson orbitals are essential to determine the deformed configurations.

Very recently, the folding model with AMD projectile density succeeded in reproducing measured $\sigma_{\text {I }}$ for ${ }^{28-30,32} \mathrm{Ne}$ by virtue of large deformation of the projectiles [36]. For ${ }^{31} \mathrm{Ne}$, the theoretical calculation underestimated measured $\sigma_{\mathrm{I}}$ by about $3 \%$. The AMD density has an inaccurate asymptotic form, since each nucleon is described by a Gaussian wave packet in AMD. The error coming from the inaccuracy is not negligible when the one-neutron separation energy $S_{n}$ is small, say $S_{n} \lesssim 1 \mathrm{MeV}$, and thereby the nucleus has halo structure [15]. The tail correction to the AMD density was then made by the ${ }^{30} \mathrm{Ne}+n$ resonating group method (RGM) with core excitations in which the deformed ground and excited states of ${ }^{30} \mathrm{Ne}$ are calculated with AMD [36]. The folding model with the tail-corrected AMD-RGM density reproduces measured $\sigma_{\text {I }}$ for ${ }^{31} \mathrm{Ne}$. The fact leads to the conclusion that ${ }^{31} \mathrm{Ne}$ is the deformed halo nucleus, which resides in the island of inversion.

The deformed Woods-Saxon (def-WS) model with AMD deformation provides the matter density with the proper asymptotic form [37]. The def-WS model well reproduces measured $\sigma_{\mathrm{R}}$ for ${ }^{20-32} \mathrm{Ne}$, and the results of the def-WS density with AMD deformation are consistent with those of the AMD density for ${ }^{20-30,32} \mathrm{Ne}$ and with that of the AMD-RGM density for ${ }^{31} \mathrm{Ne}$. The def-WS model with AMD deformation is thus a handy way of simulating AMD or AMD-RGM densities. The def-WS model with AMD deformation also reproduces measured $\sigma_{\mathrm{R}}$ for ${ }^{24-36} \mathrm{Mg}$ [18]. As another advantage of the def-WS model, one can fine tune the theoretical result to the experimental data precisely by changing the potential parameters or the deformation parameter slightly. Making this analysis for ${ }^{37} \mathrm{Mg}$, we suggested in our previous work [18] that ${ }^{37} \mathrm{Mg}$ is a deformed halo nucleus.

In this paper, we first determine matter radii of ${ }^{24-38} \mathrm{Mg}$ systematically from measured $\sigma_{\mathrm{R}}$ by fine tuning the parameters of the def-WS model. This flexibility is an advantage of the def-WS model. We next confirm that matter radii are largely enhanced by nuclear deformation for $\mathrm{Mg}$ isotopes.

Fully microscopic AMD calculations, meanwhile, have no adjustable parameter and hence high predictability, if the results of AMD calculations are consistent with existing experimental data. We then compare the deduced matter radii with the results of AMD calculations. The calculations are successful in reproducing the deduced matter radii particularly for ${ }^{24-36} \mathrm{Mg}$. For ${ }^{37,38} \mathrm{Mg}$, meanwhile, AMD calculations considerably underestimate the deduced matter radii. This sug- gests that ${ }^{37,38} \mathrm{Mg}$ are candidates for deformed halo nucleus. AMD calculations are also successful in reproducing existing experimental data on other ground-state properties (spin parity, total binding energy, and one-neutron separation energy) of $\mathrm{Mg}$ isotopes. $\mathrm{N}$ dependence of deformation parameter and neutron-skin thickness is therefore predicted with AMD.

The theoretical framework is presented in Sec. II. Numerical results are shown in Sec. III. Comparison of the def-WS results with the AMD results is made and structure of ${ }^{37} \mathrm{Mg}$ is discussed in Sec. IV. Section V is devoted to a summary.

\section{THEORETICAL FRAMEWORK}

In this section, we recapitulate the folding model, AMD, and the def-WS model. Further explanation is presented in Ref. [15] for the folding and def-WS models and Refs. [15,33] for AMD.

\section{A. Folding model}

The nucleus-nucleus scattering is governed by the manybody Schrödinger equation,

$$
\left(T_{R}+h_{\mathrm{P}}+h_{\mathrm{T}}+\sum_{i \in \mathrm{P}, j \in \mathrm{T}} v_{i j}-E\right) \Psi^{(+)}=0,
$$

with the realistic nucleon-nucleon interaction $v_{i j}$, where $E$ is the total energy, $T_{R}$ is the kinetic energy between the projectile $(\mathrm{P})$ and the target $(\mathrm{T})$, and $h_{\mathrm{P}}\left(h_{\mathrm{T}}\right)$ is the internal Hamiltonian of $\mathrm{P}(\mathrm{T})$. Equation (1) is reduced to

$$
\left(T_{R}+h_{\mathrm{P}}+h_{\mathrm{T}}+\sum_{i \in \mathrm{P}, j \in \mathrm{T}} \tau_{i j}-E\right) \hat{\Psi}^{(+)}=0
$$

by using the multiple-scattering theory [38,39] for nucleusnucleus scattering [40]. Here $\tau_{i j}$ is the effective nucleonnucleon interaction in the nuclear medium, and the $g$ matrix is often used as such $\tau_{i j}$ [19-28]. At intermediate and high incident energies of our interest, breakup and collective excitations of $\mathrm{P}$ and $\mathrm{T}$ are small [15], and Eq. (2) is further reduced to the single-channel equation

$$
\left[T_{R}+U-E_{\mathrm{in}}\right] \psi=0,
$$

with the folding potential

$$
U=\left\langle\Phi_{0}\left|\sum_{i \in \mathrm{P}, j \in \mathrm{T}} \tau_{i j}\right| \Phi_{0}\right\rangle,
$$

where $E_{\text {in }}$ is the incident energy, $\Phi_{0}$ is the product of the ground states of $\mathrm{P}$ and $\mathrm{T}$, and $\psi$ is the relative wave function between $\mathrm{P}$ and $\mathrm{T}$. This is nothing but the folding model based on the $g$-matrix interaction. In particular, the folding model with the Melbourne $g$ matrix [26] well reproduces $\sigma_{\mathrm{R}}$ for $\mathrm{Ne}$ isotopes incident on a ${ }^{12} \mathrm{C}$ target at $240 \mathrm{MeV} / \mathrm{A}$ $[15,36,37]$. 
The potential is composed of the direct and exchange parts, $U^{\mathrm{DR}}$ and $U^{\mathrm{EX}}[41,42]$ :

$$
\begin{aligned}
U^{\mathrm{DR}}(\boldsymbol{R})= & \sum_{\mu, \nu} \int \rho_{\mathrm{P}}^{\mu}\left(\boldsymbol{r}_{\mathrm{P}}\right) \rho_{\mathrm{T}}^{\nu}\left(\boldsymbol{r}_{\mathrm{T}}\right) g_{\mu \nu}^{\mathrm{DR}}\left(s ; \rho_{\mu \nu}\right) d \boldsymbol{r}_{\mathrm{P}} d \boldsymbol{r}_{\mathrm{T}}, \\
U^{\mathrm{EX}}(\boldsymbol{R})= & \sum_{\mu, \nu} \int \rho_{\mathrm{P}}^{\mu}\left(\boldsymbol{r}_{\mathrm{P}}, \boldsymbol{r}_{\mathrm{P}}-\boldsymbol{s}\right) \rho_{\mathrm{T}}^{v}\left(\boldsymbol{r}_{\mathrm{T}}, \boldsymbol{r}_{\mathrm{T}}+\boldsymbol{s}\right) \\
& \times g_{\mu \nu}^{\mathrm{EX}}\left(s ; \rho_{\mu \nu}\right) \exp [-i \boldsymbol{K}(\boldsymbol{R}) \cdot \boldsymbol{s} / M] d \boldsymbol{r}_{\mathrm{P}} d \boldsymbol{r}_{\mathrm{T}},
\end{aligned}
$$

where $\boldsymbol{R}$ is the relative coordinate between $\mathrm{P}$ and T, $\boldsymbol{s}=$ $\boldsymbol{r}_{\mathrm{P}}-\boldsymbol{r}_{\mathrm{T}}+\boldsymbol{R}$, and $\boldsymbol{r}_{\mathrm{P}}\left(\boldsymbol{r}_{\mathrm{T}}\right)$ is the coordinate of the interacting nucleon from $\mathrm{P}(\mathrm{T})$. Each of $\mu$ and $v$ denotes the $z$ component of isospin; $1 / 2$ means neutron and $-1 / 2$ does proton. The nonlocal $U^{\mathrm{EX}}$ has been localized in Eq. (6) with the local semiclassical approximation [21], where $\boldsymbol{K}(\boldsymbol{R})$ is the local momentum between $\mathrm{P}$ and $\mathrm{T}$, and $M=A A_{\mathrm{T}} /\left(A+A_{\mathrm{T}}\right)$ for the mass number $A\left(A_{\mathrm{T}}\right)$ of $\mathrm{P}(\mathrm{T})$; see Refs. $[43,44]$ for the validity of the localization. The direct and exchange parts, $g_{\mu \nu}^{\mathrm{DR}}$ and $g_{\mu \nu}^{\mathrm{EX}}$, of the $g$ matrix depend on the local density

$$
\rho_{\mu \nu}=\rho_{\mathrm{P}}^{\mu}\left(\boldsymbol{r}_{\mathrm{P}}-\boldsymbol{s} / 2\right)+\rho_{\mathrm{T}}^{\nu}\left(\boldsymbol{r}_{\mathrm{T}}+\boldsymbol{s} / 2\right)
$$

at the midpoint of the interacting nucleon pair; see Ref. [15] for the explicit forms of $g_{\mu \nu}^{\mathrm{DR}}$ and $g_{\mu \nu}^{\mathrm{EX}}$.

The relative wave function $\psi$ is decomposed into partial waves $\chi_{L}$, each with different orbital angular momentum $L$. The elastic $S$-matrix elements $S_{L}$ are obtained from the asymptotic form of the $\chi_{L}$. The total reaction cross section $\sigma_{\mathrm{R}}$ is calculable from the $S_{L}$ as

$$
\sigma_{\mathrm{R}}=\frac{\pi}{K^{2}} \sum_{L}(2 L+1)\left(1-\left|S_{L}\right|^{2}\right) .
$$

The potential $U$ has the nonspherical part when the projectile spin is nonzero. In the present calculation, we neglect the nonspherical part since the approximation is confirmed to be quite good for the reaction cross section $[15,29]$.

\section{B. AMD}

AMD starts with the many-body Schrödinger equation

$$
H=T+\sum_{i<j} \bar{v}_{i j}-T_{\mathrm{cm}}
$$

with the effective nucleon-nucleon interaction $\bar{v}_{i j}$, where the center-of-mass kinetic energy $T_{\mathrm{cm}}$ is subtracted from the kinetic energy $T$ of nucleons. In this paper, we use the GognyD1S effective nucleon-nucleon interaction [31] plus Coulomb interaction as $\bar{v}_{i j}$.

The variational wave function $\Phi^{\pi}$ is parity projected from the Slater determinant of nucleon wave packets:

$$
\Phi^{\pi}=P^{\pi} \mathcal{A}\left\{\varphi_{1}, \varphi_{2}, \ldots, \varphi_{A}\right\},
$$

where $P^{\pi}(\mathcal{A})$ is the parity-projection (antisymmetrization) operator. The $i$ th single-particle wave packet $\varphi_{i}$ is defined by $\varphi_{i}=\phi_{i}(\boldsymbol{r}) \chi_{i} \xi_{i}$ with

$$
\phi_{i}(\boldsymbol{r})=\prod_{\sigma=x, y, z}\left(\frac{2 v_{\sigma}}{\pi}\right)^{1 / 4} \exp \left\{-v_{\sigma}\left(r_{\sigma}-\frac{Z_{i \sigma}}{\sqrt{v_{\sigma}}}\right)^{2}\right\},
$$

$$
\begin{aligned}
& \chi_{i}=\alpha_{i, \uparrow} \chi_{\uparrow}+\alpha_{i, \downarrow} \chi_{\downarrow}, \quad\left|\alpha_{i, \uparrow}\right|^{2}+\left|\alpha_{i, \downarrow}\right|^{2}=1, \\
& \xi_{i}=p \text { or } n .
\end{aligned}
$$

The centroids $\boldsymbol{Z}_{i}$, the width $v_{\sigma}$, and the spin directions $\alpha_{i, \uparrow}$ and $\alpha_{i, \downarrow}$ of Gaussian wave packets are variational parameters. The center-of-mass wave function can be analytically separated from the variational wave function. Hence all quantities calculated with AMD are free from the spurious center-ofmass motion.

The parameters in Eq. (10) are determined with the frictional cooling method by minimizing the total energy under the constraint on the matter quadrupole deformation parameter $\bar{\beta}$ defined by

$$
\begin{aligned}
& \frac{\left\langle x^{2}\right\rangle^{1 / 2}}{\left[\left\langle x^{2}\right\rangle\left\langle y^{2}\right\rangle\left\langle z^{2}\right\rangle\right]^{1 / 6}}=\exp \left[\sqrt{\frac{5}{4 \pi}} \bar{\beta} \cos \left(\bar{\gamma}+\frac{2 \pi}{3}\right)\right], \\
& \frac{\left\langle y^{2}\right\rangle^{1 / 2}}{\left[\left\langle x^{2}\right\rangle\left\langle y^{2}\right\rangle\left\langle z^{2}\right\rangle\right]^{1 / 6}}=\exp \left[\sqrt{\frac{5}{4 \pi}} \bar{\beta} \cos \left(\bar{\gamma}-\frac{2 \pi}{3}\right)\right], \\
& \frac{\left\langle z^{2}\right\rangle^{1 / 2}}{\left[\left\langle x^{2}\right\rangle\left\langle y^{2}\right\rangle\left\langle z^{2}\right\rangle\right]^{1 / 6}}=\exp \left[\sqrt{\frac{5}{4 \pi}} \bar{\beta} \cos \bar{\gamma}\right] .
\end{aligned}
$$

Here, $\left\langle x^{2}\right\rangle,\left\langle y^{2}\right\rangle$, and $\left\langle z^{2}\right\rangle$ defined in the intrinsic frame are so chosen to satisfy the ordering $\left\langle x^{2}\right\rangle \leqslant\left\langle y^{2}\right\rangle \leqslant\left\langle z^{2}\right\rangle$. Since no constraint is imposed on $\bar{\gamma}$, it has an optimal value for each value of $\bar{\beta}$. $\bar{\beta}$,

After the variation, we perform the AMP for each value of

$$
\begin{aligned}
\Phi_{m K}^{I \pi}(\bar{\beta}) & =P_{m K}^{I} \Phi_{\mathrm{int}}^{\pi}(\bar{\beta}), \\
P_{m K}^{I} & =\frac{2 I+1}{8 \pi^{2}} \int d \Omega D_{m K}^{I *}(\Omega) R(\Omega),
\end{aligned}
$$

where $D_{m K}^{I}(\Omega)$ and $R(\Omega)$ are the Wigner's $D$ function and the rotation operator, respectively. The wave functions that have the same parity and angular momentum $(I, m)$ are superposed as

$$
\Phi_{n}^{I m \pi}=\sum_{K=-I}^{I} \sum_{\bar{\beta}} c_{n K}(\bar{\beta}) \Phi_{m K}^{I \pi}(\bar{\beta}),
$$

where $\bar{\beta}$ is varied from 0 to 1 with an interval of 0.025 in actual calculations. The coefficients $c_{n K}(\bar{\beta})$ are determined by solving the Hill-Wheeler equation.

The ground-state wave function $\Phi_{\text {g.s. }}^{I m \pi}$ thus obtained is transformed into the nucleon density as

$$
\begin{aligned}
\rho_{I m I m^{\prime}}(\boldsymbol{r}) & =\left\langle\Phi_{\mathrm{g} . \mathrm{s} .}^{I m \pi}\left|\sum_{i} \delta\left(\boldsymbol{r}_{i}-\boldsymbol{X}-\boldsymbol{r}\right)\right| \Phi_{\mathrm{g} . \mathrm{I} .}^{I m^{\prime} \pi}\right\rangle, \\
& =\sum_{\lambda=0}^{2 I} \rho_{I I}^{(\lambda)}(r)\left(\operatorname{Im}{ }^{\prime} \lambda \mu \mid \operatorname{Im}\right) Y_{\lambda \mu}^{*}(\hat{\boldsymbol{r}}),
\end{aligned}
$$

where $X$ denotes the center-of-mass coordinate. When $I>$ 0 , the multipolarity $\lambda$ can take nonzero values. The nonzero components make the folding potential $U$ nonspherical. But the effects are small on $\sigma_{\mathrm{R}}[15,29]$. We then take only the spherical $(\lambda=0)$ component in this paper. 


\section{C. def-WS model} parts:

The def-WS potential consists of the central and spin-orbit

$$
\begin{aligned}
V_{\mathrm{c}}(\boldsymbol{r}) & =\frac{V_{0}}{1+\exp \left[\operatorname{dist}_{\Sigma}(\boldsymbol{r}) / a\right]}, \\
V_{\mathrm{so}}(\boldsymbol{r}) & =\lambda_{\mathrm{so}}\left(\frac{\hbar}{2 m_{\mathrm{red}} c}\right)^{2} \nabla V_{\mathrm{c}}(\boldsymbol{r}) \cdot\left(\boldsymbol{\sigma} \times \frac{1}{i} \nabla\right),
\end{aligned}
$$

where $m_{\text {red }}=m(A-1) / A$ for nucleon mass $m$ and the function $\operatorname{dist}_{\Sigma}(\boldsymbol{r})$ represents the distance of a point $\boldsymbol{r}$ from the deformed surface $\Sigma$ that is specified by the radius

$$
R(\theta, \phi ; \boldsymbol{\alpha})=R_{0} c_{v}(\boldsymbol{\alpha})\left[1+\sum_{\lambda \mu} \alpha_{\lambda \mu}^{*} Y_{\lambda \mu}(\theta, \phi)\right]
$$

with the deformation parameters $\boldsymbol{\alpha} \equiv\left\{\alpha_{\lambda \mu}\right\}$. The constant $c_{v}(\boldsymbol{\alpha})$ is introduced to guarantee the volume conservation of nucleus. Since the effect of the hexadecapole deformation on the reaction cross section is rather small [15], we only include the quadrupole deformation in this study. The parameter set $\left(\alpha_{2 \mu}\right)$ is related to the standard set $\left(\beta_{2}, \gamma\right)$ as

$$
\begin{aligned}
& \alpha_{20}=\beta_{2} \cos \gamma, \\
& \alpha_{22}=\alpha_{2-2}=-\frac{1}{\sqrt{2}} \beta_{2} \sin \gamma .
\end{aligned}
$$

As the parameter set $\left(V_{0}, R_{0}, a, \lambda_{\text {so }}\right)$ of the Woods-Saxon (WS) potential, we take a recent parametrization given by Wyss [45]; see Appendix A for actual values of the parameter set.

The deformation parameters $\left(\beta_{2}, \gamma\right)$ in the def-WS model are determined from the corresponding deformation parameters $(\bar{\beta}, \bar{\gamma})$ in AMD. Here, the parameters $(\bar{\beta}, \bar{\gamma})$ of AMD wave function $\Phi_{n}^{I m \pi}$ [Eq. (19)] are defined as those of the basis wave function $\Phi_{m K}^{I \pi}(\bar{\beta})$ [Eq. (17)], which has the maximum overlap with $\Phi_{n}^{I m \pi}$. The relation between $(\bar{\beta}, \bar{\gamma})$ in AMD and $\left(\beta_{2}, \gamma\right)$ in the def-WS model is obtained so that both the models can yield the same ratio $\left\langle x^{2}\right\rangle:\left\langle y^{2}\right\rangle:\left\langle z^{2}\right\rangle$; see Appendix B for further explanation and actual values of $\bar{\beta}$ and $\beta_{2}$ for $\mathrm{Mg}$ isotopes.

The nucleon density calculated by the def-WS model is the intrinsic density in the body-fixed frame and unisotropic, while the density used in the folding model is that in the laboratory frame. In order to obtain the latter from the former one has to perform the AMP. Instead, we use the angle average of the deformed intrinsic density, which has been confirmed to be a good approximation of the projected density; see Ref. [15] for details.

\section{Spherical HF and HFB}

As a reference, the spherical Hartree-Fock (HF) and spherical Hartree-Fock-Bogoliubov (HFB) methods are employed to calculate the nucleon density for the spherical systems. In the HF and HFB calculations it is important to properly choose the effective interaction in order to obtain accurate description of the ground state. We use consistently the same Gogny-D1S interaction [31] as in the AMD calculation for this purpose. We refer to the spherical Gogny HF and HFB methods as sph-GHF and sph-GHFB in this paper. The spherical shape is imposed with the filling approximation as a standard manner, whenever

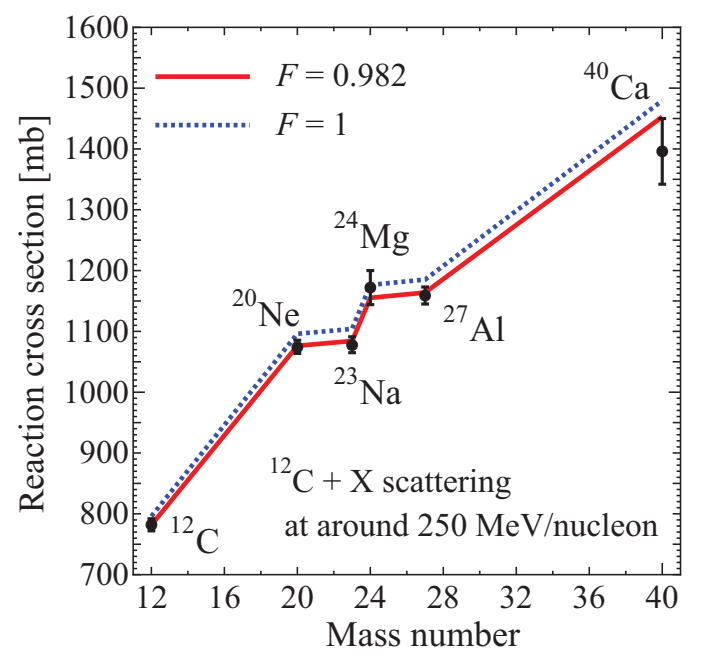

FIG. 1. (Color online) Reaction cross sections for the ${ }^{12} \mathrm{C}$ scattering on stable nuclei from $A=12$ to 40 . The data for ${ }^{12} \mathrm{C}$ and ${ }^{27} \mathrm{Al}$ at $250.8 \mathrm{MeV} /$ nucleon are taken from Ref. [48]. The data for ${ }^{20} \mathrm{Ne}$ and ${ }^{23} \mathrm{Na}$ at $250 \mathrm{MeV} /$ nucleon are deduced from the measured $\sigma_{\mathrm{I}}$ at around $1 \mathrm{GeV} /$ nucleon [49,50] with the Glauber model [17]. The data for ${ }^{40} \mathrm{Ca}$ at $240 \mathrm{MeV} /$ nucleon is obtained from the measured $\sigma_{\mathrm{R}}$ at $83 \mathrm{MeV} /$ nucleon [51] with the Glauber model [18]. The solid (dotted) line stands for the results of the folding-model calculations after (before) the normalization with a factor $F=0.982$.

sph-GHF and sph-GHFB calculations are done. In actual sph-GHF and sph-GHFB calculations, we adopt the Gaussian expansion method [46], which reduces numerical tasks.

\section{RESULTS}

\section{A. Reaction cross sections for stable nuclei}

First, the accuracy of the Melbourne $g$-matrix folding model (i.e., the folding model with the Melbourne $g$-matrix interaction) is tested for the scattering of ${ }^{12} \mathrm{C}$ from several stable nuclei at intermediate energies around $250 \mathrm{MeV} /$ nucleon. The $g$-matrix folding model was successful in reproducing measured reaction cross sections for ${ }^{12} \mathrm{C},{ }^{20} \mathrm{Ne},{ }^{23} \mathrm{Na}$, and ${ }^{27} \mathrm{Al}$ $[15,36,37]$. In this paper, we newly consider ${ }^{24} \mathrm{Mg}$ and ${ }^{40} \mathrm{Ca}$ targets in addition to the stable targets mentioned above. We employ the phenomenological densities [47] as the projectile and target densities, where the proton density is deduced from the electron scattering and the neutron distribution is assumed to have the same geometry as the proton one. This assumption is good for the stable nuclei, since the neutron rms radii are almost the same as the proton ones in sph-GHF calculations.

Figure 1 shows reaction cross sections for the ${ }^{12} \mathrm{C}$ scattering from ${ }^{12} \mathrm{C},{ }^{20} \mathrm{Ne},{ }^{23} \mathrm{Na},{ }^{24} \mathrm{Mg},{ }^{27} \mathrm{Al}$, and ${ }^{40} \mathrm{Ca}$ targets at around $250 \mathrm{MeV} /$ nucleon. The experimental data are taken from Refs. [18,48-51]. The results of the folding-model (dotted line) well reproduce the experimental data. More precisely, they slightly overestimate the data. We then introduce the normalization factor of $F=0.982$ to reproduce the mean value of the experimental data for ${ }^{12} \mathrm{C}$. The result (solid line) is consistent with experimental data for other targets. This fine tuning is taken also for the scattering of $\mathrm{Mg}$ isotopes from ${ }^{12} \mathrm{C}$ targets around $240 \mathrm{MeV} /$ nucleon. 


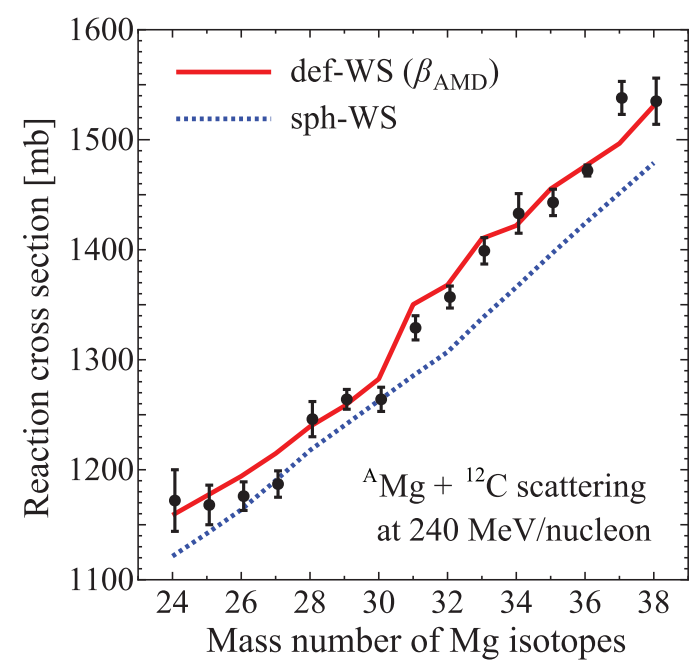

FIG. 2. (Color online) Reaction cross sections for the scattering of $\mathrm{Mg}$ isotopes at $240 \mathrm{MeV} /$ nucleon. The experimental data are taken from Ref. [18]. The solid line stands for the results of the def-WS model with AMD deformation, whereas the dotted line corresponds to the results of spherical Woods-Saxon (sph-WS) calculations.

\section{B. Matter radii of $\mathrm{Mg}$ isotopes}

Figure 2 shows a comparison of calculated and measured $\sigma_{\mathrm{R}}$ for the scattering of ${ }^{24-38} \mathrm{Mg}$ on a ${ }^{12} \mathrm{C}$ target at $240 \mathrm{MeV} /$ nucleon. The $\sigma_{\mathrm{R}}$ are evaluated by the folding model with different types of projectile densities; one is the densities calculated by the def-WS model with AMD deformation and the other is the densities of spherical Woods-Saxon (sph-WS) calculations. The solid line denotes the results of the def-WS model, whereas the dotted line corresponds to the results of sph-WS calculations. The large difference between the two lines shows that nuclear deformation effects are important in $\sigma_{\mathrm{R}}$. The def-WS model yields good agreement with the experimental data [18]. For ${ }^{37} \mathrm{Mg}$, however, the def-WS model slightly underestimates the measured $\sigma_{\mathrm{R}}$, indicating that ${ }^{37} \mathrm{Mg}$ is a deformed halo nucleus; see Ref. [18] and Sec. IV B for details.

Since the def-WS results are consistent with the measured $\sigma_{\mathrm{R}}$, we deduce the rms radii of $\mathrm{Mg}$ isotopes from the measured $\sigma_{\mathrm{R}}$ by fitting it with the calculation, where either the depth parameter $V_{0}$ or the deformation parameter $\beta_{2}$ are adjusted slightly in the def-WS potential. This flexibility is a merit of the def-WS model.

The relation between $\sigma_{\mathrm{R}}$ and the corresponding rms matter radius is plotted in Fig. 3 for two nuclei; Fig. 3(a) ${ }^{24} \mathrm{Mg}$ and Fig. 3(b) ${ }^{37} \mathrm{Mg}$. The closed circles denote the results of the defWS model in which $\beta_{2}$ is varied from 0 to 0.6 with the interval of 0.1 with keeping all other parameters. Note that the AMD deformation used in Fig. 3 is $\beta_{2}=0.433$ for ${ }^{24} \mathrm{Mg}$ and is $\beta_{2}=0.362$ for ${ }^{37} \mathrm{Mg}$, respectively (see Table IV). Larger $\beta_{2}$ yields larger rms radius and hence larger $\sigma_{\mathrm{R}}$. The open squares correspond to the results of the def-WS model in which $\left|V_{0}\right|$ is reduced by a factor $1 \sim 0.72$ with interval 0.04 for ${ }^{24} \mathrm{Mg}$ in Fig. 3(a) and by a factor $1 \sim 0.92$ with interval 0.01 for ${ }^{37} \mathrm{Mg}$ in Fig. 3(b). The results of sph-GHF, spherical Woods-Saxon (sph-WS), and AMD densities are also shown by open triangles
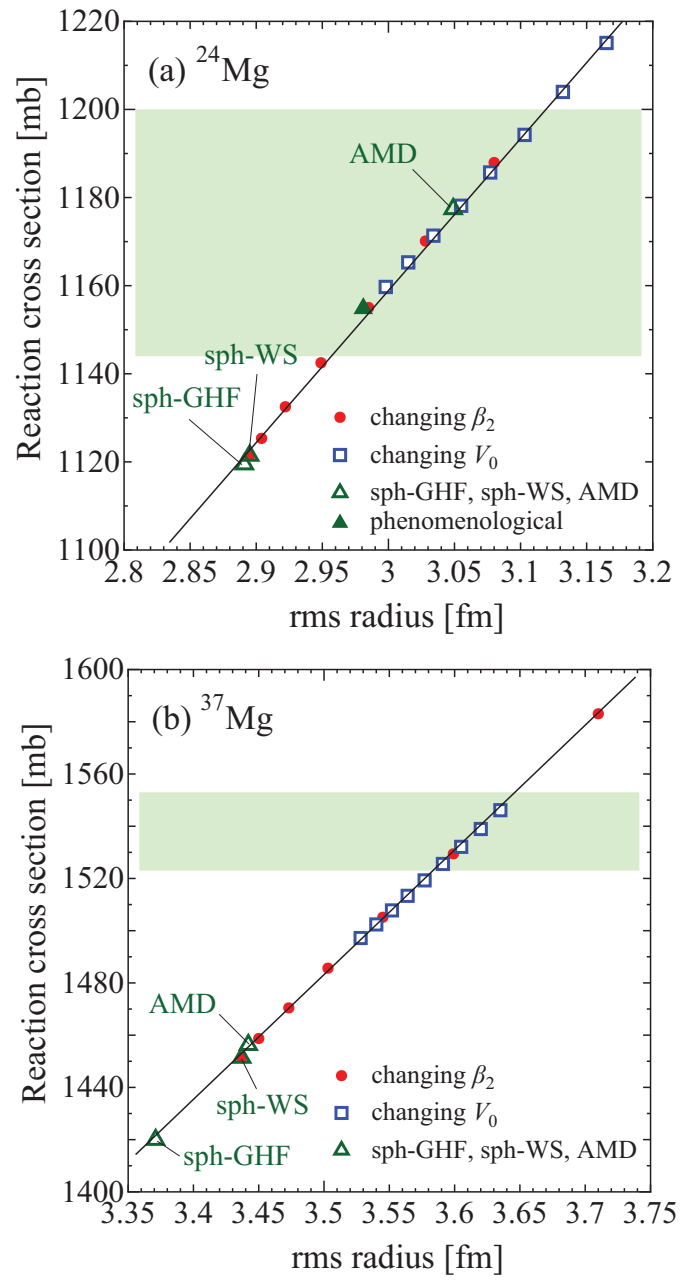

FIG. 3. (Color online) Relation between the rms matter radius and the reaction cross section for (a) ${ }^{24} \mathrm{Mg}$ and (b) ${ }^{37} \mathrm{Mg}$. The closed circles stands for the results of the def-WS model in which $\beta_{2}$ is varied from 0 to 0.6 with interval 0.1 , and the open squares correspond to the results of the def-WS model in which $\left|V_{0}\right|$ is reduced by a factor $1 \sim 0.72$ with interval 0.04 for ${ }^{24} \mathrm{Mg}$ in (a) and by a factor $1 \sim 0.92$ with interval 0.01 for ${ }^{37} \mathrm{Mg}$ in (b). Smaller $\beta_{2}$ corresponds to smaller rms matter radius and hence smaller reaction cross section, while smaller $\left|V_{0}\right|$ does to larger rms matter radius and hence larger reaction cross section. The open triangles denote the results of sphGHF, sph-WS, AMD densities from bottom. For ${ }^{24} \mathrm{Mg}$, the result of the phenomenological density [47] is also presented by a closed triangle. The hatching region shows the lower and upper bounds of measured $\sigma_{\mathrm{R}}$.

from bottom (i.e., they are in increasing order). For ${ }^{24} \mathrm{Mg}$, the result of the phenomenological density deduced from the electron scattering [47] is also presented by a closed triangle. As an important result, all the results are on a straight line for each case of Fig. 3(a) ${ }^{24} \mathrm{Mg}$ and of Fig. 3(b) ${ }^{37} \mathrm{Mg}$. We can then precisely determine matter radii of $\mathrm{Mg}$ isotopes corresponding to the measured $\sigma_{\mathrm{R}}$ by using the straight line.

The resultant matter radii of $\mathrm{Mg}$ isotopes are tabulated in Table I and are plotted as a function of mass number $A$ in Fig. 4. The dotted and dashed lines represent the results of sph-GHF calculations for $\mathrm{Mg}$ isotopes and stable 
TABLE I. Matter radii of Mg isotopes deduced from measured $\sigma_{\mathrm{R}}$. Mean values of deduced matter radii are evaluated from those of the corresponding $\sigma_{\mathrm{R}}$, while errors of deduced matter radii are estimated from those of measured $\sigma_{\mathrm{R}}$ by the straight solid lines shown in Fig. 3. All the values are shown in units of $\mathrm{fm}$.

\begin{tabular}{lcc}
\hline \hline nuclide & rms radius & error \\
\hline${ }^{24} \mathrm{Mg}$ & 3.03 & 0.08 \\
${ }^{25} \mathrm{Mg}$ & 2.99 & 0.05 \\
${ }^{26} \mathrm{Mg}$ & 2.99 & 0.04 \\
${ }^{27} \mathrm{Mg}$ & 2.99 & 0.03 \\
${ }^{28} \mathrm{Mg}$ & 3.12 & 0.04 \\
${ }^{29} \mathrm{Mg}$ & 3.14 & 0.02 \\
${ }^{30} \mathrm{Mg}$ & 3.11 & 0.03 \\
${ }^{31} \mathrm{Mg}$ & 3.25 & 0.03 \\
${ }^{32} \mathrm{Mg}$ & 3.30 & 0.02 \\
${ }^{33} \mathrm{Mg}$ & 3.38 & 0.03 \\
${ }^{34} \mathrm{Mg}$ & 3.44 & 0.04 \\
${ }^{35} \mathrm{Mg}$ & 3.44 & 0.03 \\
${ }^{36} \mathrm{Mg}$ & 3.49 & 0.01 \\
${ }^{37} \mathrm{Mg}$ & 3.62 & 0.03 \\
${ }^{38} \mathrm{Mg}$ & 3.60 & 0.04 \\
\hline \hline
\end{tabular}

$A=24-40$ nuclei, respectively. The two lines correspond to matter radii of the nuclei in the spherical limit. For the spherical nucleus ${ }^{40} \mathrm{Ca}$, the matter radius determined from the electron scattering [47] are plotted by an open triangle, which lies on the dashed line, indicating the reliability of sph-GHF calculations. The difference between the dotted and dashed lines shows the neutron-skin effects; the increase of neutron excess makes the Fermi energy of neutrons much larger than that of protons, and consequently the neutron radius bulges out

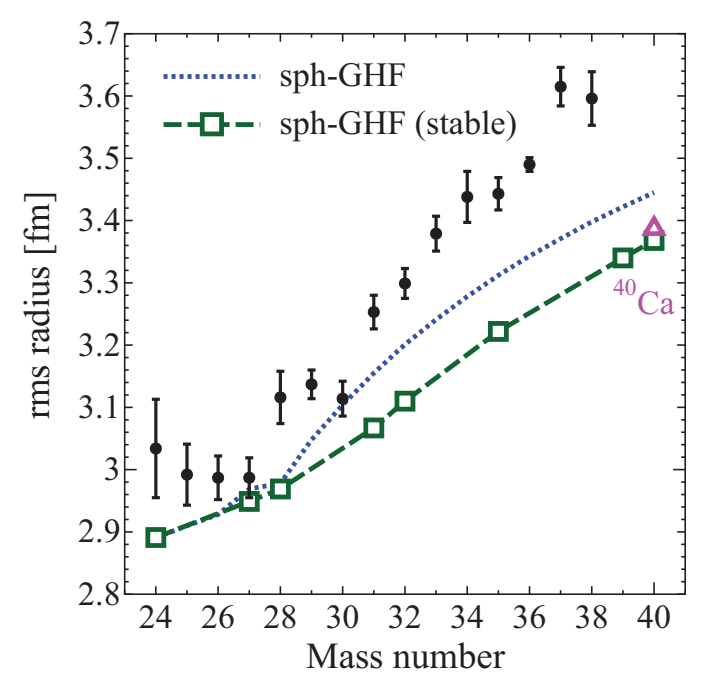

FIG. 4. (Color online) Matter radii of Mg isotopes deduced from measured $\sigma_{R}$. The dotted line denotes the results of sph-GHF calculations. The calculated radii by the sph-GHF model for stable $A=24-40$ nuclei are also included as the dashed line, where the nuclei included are ${ }^{24} \mathrm{Mg},{ }^{27} \mathrm{Al},{ }^{28} \mathrm{Si},{ }^{31} \mathrm{P},{ }^{32} \mathrm{~S},{ }^{35} \mathrm{Cl},{ }^{39} \mathrm{~K}$, and ${ }^{40} \mathrm{Ca}$. For spherical nucleus ${ }^{40} \mathrm{Ca}$, matter radius determined from the electron scattering [47] is shown by an open triangle. compared to those of stable $N \approx Z$ nuclei. Large enhancement of the deduced radii from the dotted line mainly comes from nuclear deformation. Further discussion is made in the next Sec. III C.

For ${ }^{37} \mathrm{Mg}$, the matter radius should be carefully deduced from the measured $\sigma_{\mathrm{R}}$, since the def-WS model with AMD deformation considerably underestimates the measured $\sigma_{\mathrm{R}}$. In the previous analysis [18], we assumed that ${ }^{37} \mathrm{Mg}$ is a deformed halo nucleus and reduced the potential depth only for the last neutron without changing the potential for the core nucleons. At $\beta_{2}=0.362$ calculated with AMD, the [312 5/2] orbital coming from the spherical $0 f_{7 / 2}$ orbital has slightly lower energy than the [321 $1 / 2]$ orbital coming from the spherical $1 p_{3 / 2}$ orbital. In the case that the last neutron is in the [312 $5 / 2$ ] orbital, the calculated $\sigma_{\mathrm{R}}$ cannot reproduce the measured $\sigma_{\mathrm{R}}$ even if the potential depth is reduced. This problem can be solved if the last neutron is in the [321 1/2] orbital. In the previous analysis, therefore, the last neutron is assumed to be in the [321 1/2] orbital and the potential depth is reduced only for the last neutron. In the present analysis, meanwhile, we simply consider that the last neutron is in the [312 5/2] orbital and reduce the potential depth uniformly for all the nucleons, i.e., the core $\left({ }^{36} \mathrm{Mg}\right)$ is also slightly expanded by the reduction. The deduced matter radius is $3.62 \pm 0.03 \mathrm{fm}$ in the present analysis and $3.65_{-0.05}^{+0.09} \mathrm{fm}$ in the previous analysis, although in the previous analysis the solid line shown in Fig. 3(b) was slightly bent for both are consistent with each other within the error bars. The matter radius is thus almost independent of the deduction procedure taken.

In Fig. 3(b), the matter radius of ${ }^{37} \mathrm{Mg}$ calculated by the sph-WS model is considerably larger than that by the sph-GHF model. This indicates that the depth of the present parametrization of the WS potential is a bit too shallow for such an unstable nucleus with large neutron excess. This point will be discussed later in Sec. IV A.

TABLE II. Ground-state properties of $\mathrm{Mg}$ isotopes predicted by AMD. For ${ }^{40} \mathrm{Mg}$, the two-neutron separation energy $S_{-2 n}$ is shown, since ${ }^{39} \mathrm{Mg}$ is unbound in AMD calculations.

\begin{tabular}{lcclll}
\hline \hline nuclide & $I^{\pi}(\exp )$ & $I^{\pi}(\mathrm{AMD})$ & $S_{-1 n}[\mathrm{MeV}]$ & $\bar{\beta}$ & $\bar{\gamma}$ \\
\hline${ }^{24} \mathrm{Mg}$ & $0^{+}$ & $0^{+}$ & & 0.42 & $0^{\circ}$ \\
${ }^{25} \mathrm{Mg}$ & $5 / 2^{+}$ & $5 / 2^{+}$ & 7.125 & 0.40 & $0^{\circ}$ \\
${ }^{26} \mathrm{Mg}$ & $0^{+}$ & $0^{+}$ & 10.211 & 0.375 & $0^{\circ}$ \\
${ }^{27} \mathrm{Mg}$ & $1 / 2^{+}$ & $1 / 2^{+}$ & 6.444 & 0.35 & $0^{\circ}$ \\
${ }^{28} \mathrm{Mg}$ & $0^{+}$ & $0^{+}$ & 8.881 & 0.35 & $0^{\circ}$ \\
${ }^{29} \mathrm{Mg}$ & $3 / 2^{+}$ & $3 / 2^{+}$ & 4.123 & 0.295 & $0^{\circ}$ \\
${ }^{30} \mathrm{Mg}$ & $0^{+}$ & $0^{+}$ & 5.781 & 0.285 & $25^{\circ}$ \\
${ }^{31} \mathrm{Mg}$ & $1 / 2^{+}$ & $1 / 2^{+}$ & 2.624 & 0.44 & $0^{\circ}$ \\
${ }^{32} \mathrm{Mg}$ & $0^{+}$ & $0^{+}$ & 5.598 & 0.395 & $0^{\circ}$ \\
${ }^{33} \mathrm{Mg}$ & $3 / 2^{-}$ & $3 / 2^{-}$ & 2.640 & 0.44 & $0^{\circ}$ \\
${ }^{34} \mathrm{Mg}$ & $0^{+}$ & $0^{+}$ & 3.622 & 0.35 & $0^{\circ}$ \\
${ }^{35} \mathrm{Mg}$ & $\left(7 / 2^{-}\right)$ & $3 / 2^{+}$ & 1.011 & 0.40 & $0^{\circ}$ \\
${ }^{36} \mathrm{Mg}$ & $0^{+}$ & $0^{+}$ & 2.993 & 0.39 & $0^{\circ}$ \\
${ }^{37} \mathrm{Mg}$ & $\left(7 / 2^{-}\right)$ & $5 / 2^{-}$ & 0.489 & 0.355 & $0^{\circ}$ \\
${ }^{38} \mathrm{Mg}$ & $0^{+}$ & $0^{+}$ & 2.112 & 0.38 & $0^{\circ}$ \\
${ }^{39} \mathrm{Mg}$ & & & unbound & & \\
${ }^{40} \mathrm{Mg}$ & $0^{+}$ & $0^{+}$ & $1.119\left(S_{-2 n}\right)$ & 0.41 & $0^{\circ}$ \\
\hline \hline
\end{tabular}




\section{AMD analyses for Mg isotopes}

First, the ground-state properties, i.e., spin parity $I^{\pi}$, oneneutron separation energy $S_{-1 n}$, and deformation parameters $\bar{\beta}$ and $\bar{\gamma}$, of Mg isotopes are predicted by AMD and tabulated in Table II. AMD calculations yield the same $I^{\pi}$ as the data [52] for ${ }^{24-34} \mathrm{Mg}$. For ${ }^{35,37,39} \mathrm{Mg}$, meanwhile, we cannot make definite discussion on $I^{\pi}$, since it is not established experimentally. For ${ }^{37} \mathrm{Mg}$, AMD calculations yield a small $S_{-1 n}$ value consistent with the empirical values $0.16 \pm 0.68 \mathrm{MeV}$ [52], though the error is large. For ${ }^{39} \mathrm{Mg}, S_{-1 n}$ is negative in both the AMD calculation and the empirical value [52]. In fact, ${ }^{39} \mathrm{Mg}$ is experimentally shown to be unbound in Ref. [53].

In Fig. 5, total binding energy per nucleon and $S_{-1 n}$ are shown as a function of $A$ for $\mathrm{Mg}$ isotopes. The experimental data [52] are compared with the results of sph-GHF, sphGHFB, and AMD calculations, where the Gogny-D1S force is
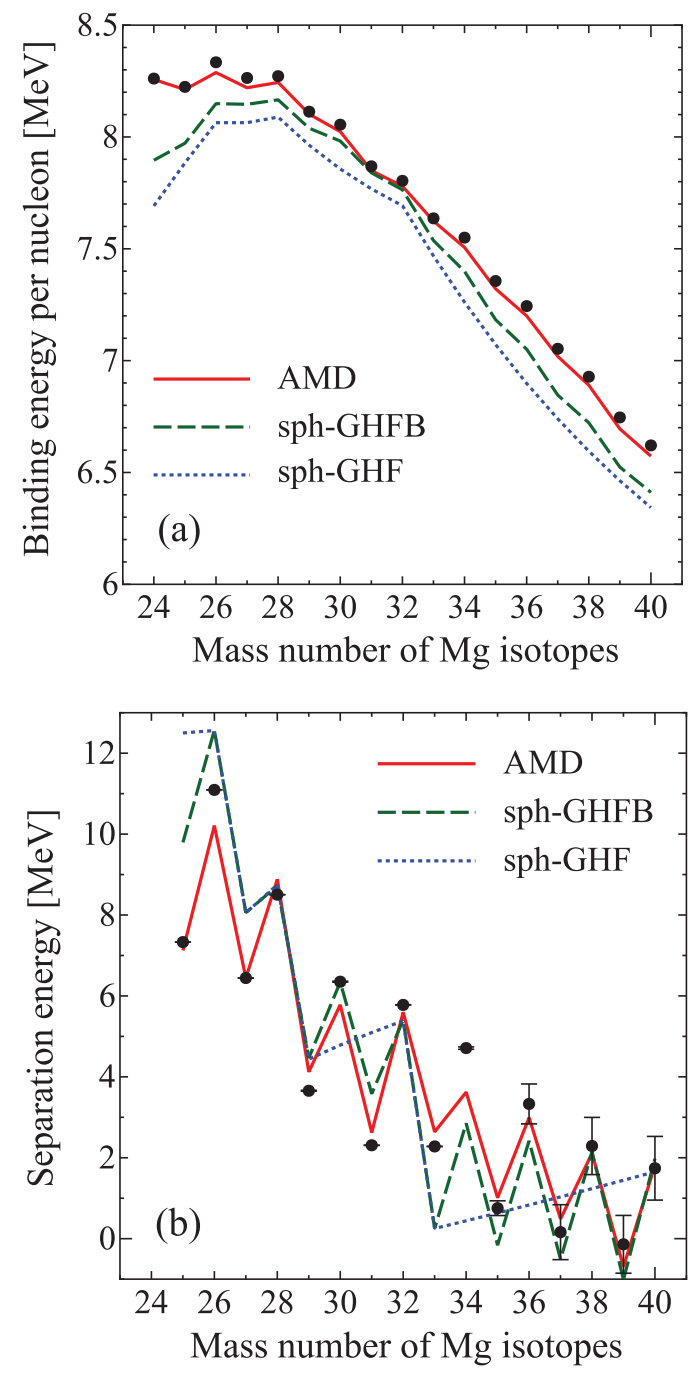

FIG. 5. (Color online) (a) Total binding energy per nucleon and (b) one-neutron separation energy as a function of mass number for $\mathrm{Mg}$ isotopes. The solid, dashed, and dotted lines represent the results of AMD, sph-GHFB, and sph-GHF calculations, respectively. ${ }^{39} \mathrm{Mg}$ is unbound in AMD calculations. The experimental data are taken from Ref. [52]. used consistently in all models. The sph-GHF results (dotted line) underestimate measured binding energies systematically and do not explain the measured odd-even staggering of $S_{-1 n}$. These are improved by sph-GHFB calculations with pairing correlations (dashed line), though $S_{-1 n}$ is negative for ${ }^{35,37,39} \mathrm{Mg}$ in the calculations. Meanwhile, AMD calculations (solid line) well reproduce the trend of the experimental data for both total binding energy and $S_{-1 n}$. The drip line of $\mathrm{Mg}$ is located at ${ }^{40} \mathrm{Mg}$ and ${ }^{39} \mathrm{Mg}$ is unbound in AMD calculations. Since the basis wave functions with different configurations around the Fermi surface are superposed in Eq. (19), the dominant effect of the pairing correlation is included in AMD calculations, as confirmed from the reasonable reproduction of the even-odd staggering in $S_{-1 n}$. Mg isotopes thus greatly gain the total energies by deformation.

In Fig. 6, matter radius and $\sigma_{\mathrm{R}}$ are plotted as a function of $A$ for $\mathrm{Mg}$ isotopes. The same discussion can be made between the two quantities. The AMD results (solid line) yield
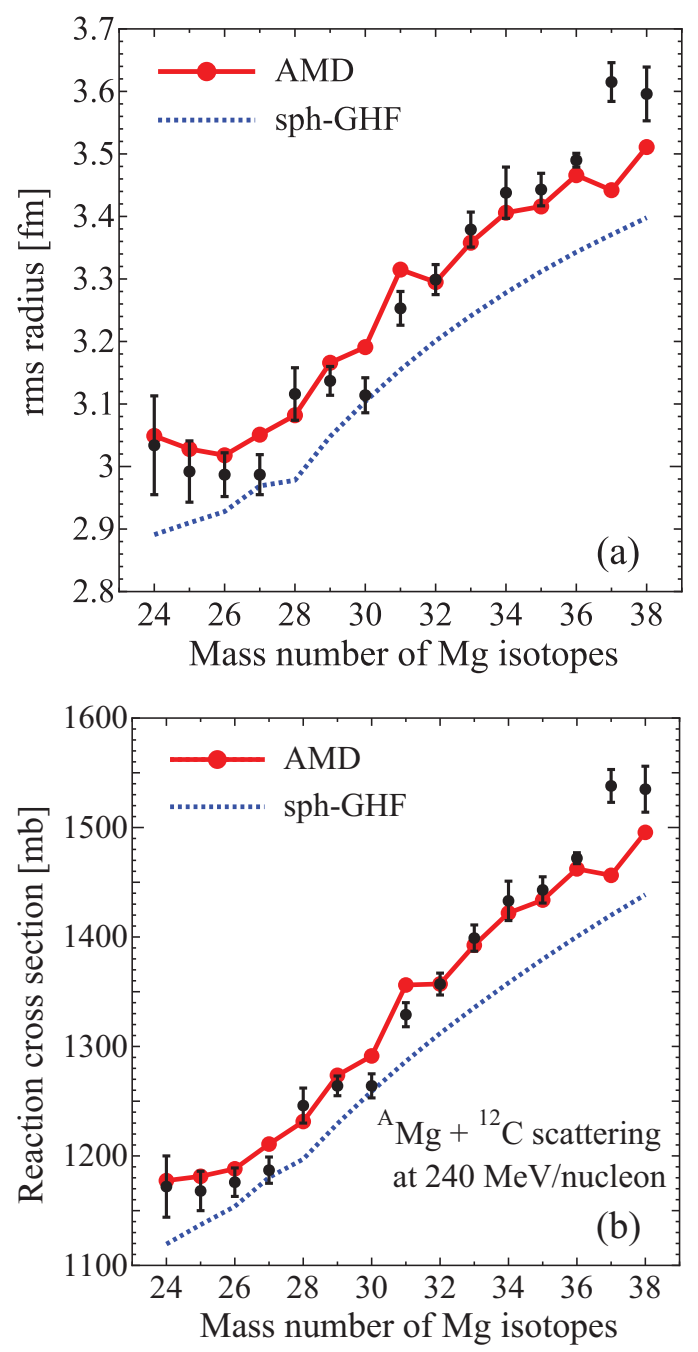

FIG. 6. (Color online) (a) Matter radii and (b) reaction cross sections $\sigma_{\mathrm{R}}$ as a function of mass number for $\mathrm{Mg}$ isotopes. The solid and dotted lines represent the results of AMD and sph-GHF calculations, respectively. The experimental data of $\sigma_{\mathrm{R}}$ are taken from Ref. [18]. 


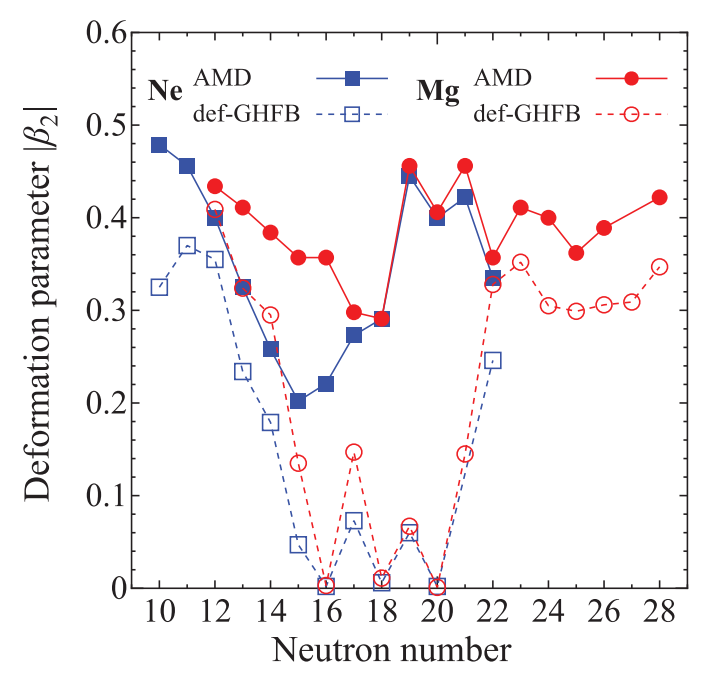

FIG. 7. (Color online) Theoretical prediction on $\left|\beta_{2}\right|$ for Mg and $\mathrm{Ne}$ isotopes. The solid line with closed circles (squares) are the AMD results with the AMP for $\mathrm{Mg}(\mathrm{Ne})$ isotopes, whereas the dashed line with open circles (squares) are the deformed Gogny-HFB (defGHFB) results of Ref. [55,56] with no AMP for $\mathrm{Mg}(\mathrm{Ne})$ isotopes.

much better agreement with the data than the sph-GHF results (dotted line); note that the effect of pairing correlations is not large for the matter radius and $\sigma_{\mathrm{R}}$, and the sph-GHFB results agree with the sph-GHF results within the thickness of line. Deformation enhances matter radius and $\sigma_{\mathrm{R}}$ from the dotted to the solid line, indicating the importance of deformation on matter radius. The AMD results reproduce the data for ${ }^{24-36} \mathrm{Mg}$, although it considerably underestimates the data for ${ }^{37,38} \mathrm{Mg}$. Thus AMD predicts ground-state properties of $\mathrm{Mg}$ isotopes properly. However, an exception is the underestimation of matter radius for ${ }^{37,38} \mathrm{Mg}$. This will be discussed in Sec. IV B.

In Fig. 7, the absolute value of $\beta_{2}$ is plotted as a function of neutron number $N$ for $\mathrm{Mg}$ and $\mathrm{Ne}$ isotopes. The solid line with closed circles (squares) stands for the AMD results for $\mathrm{Mg}(\mathrm{Ne})$ isotopes; the values for $\mathrm{Ne}$ isotopes are taken from Ref. [15]. For both $\mathrm{Mg}$ and $\mathrm{Ne}$ isotopes, the AMD results show an abrupt increase of $\left|\beta_{2}\right|$ when $N$ varies from 18 to 19 , where the Nilsson orbitals originating from the spherical $0 f_{7 / 2}$ shell start to be occupied [33]. This indicates that the island of inversion starts at $N=19$ and the $N=20$ magicity disappears. At $N=19 \sim 28$, the $\left|\beta_{2}\right|$ keep large values of around 0.4 . Thus we cannot identify the endpoint of the island of inversion. This statement is consistent with the result of in-beam $\gamma$-ray spectroscopy of ${ }^{34,36,38} \mathrm{Mg}$ [54] that the deduced $E\left(4^{+}\right) / E\left(2^{+}\right)$ratios are about 3.1 independently of $N$. The $N=28$ magicity, moreover, disappears, since $\beta_{2}$ is large for ${ }^{40} \mathrm{Mg}$. The fact that $\left|\beta_{2}\right|$ is large from ${ }^{31} \mathrm{Mg}$ with $N=19$ to a drip-line nucleus ${ }^{40} \mathrm{Mg}$ with $N=28$ indicates that the socalled island of inversion may not be an island but a peninsula reaching the drip line.

Now the AMD results are compared with the deformed Gogny-HFB (def-GHFB) results of Refs. [55,56] in Fig. 7, where the dashed line with open circles (squares) denote the def-GHFB results for $\mathrm{Mg}(\mathrm{Ne})$ isotopes. Note that the AMP

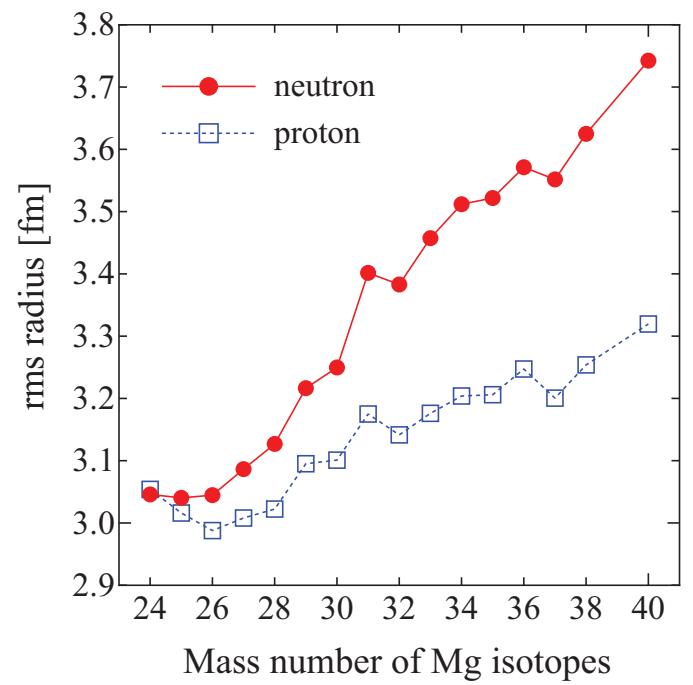

FIG. 8. (Color online) AMD prediction on neutron and proton rms radii for $\mathrm{Mg}$ isotopes. The solid (dashed) line denotes the neutron (proton) radius.

is performed in AMD but not in the def-GHFB calculations of Refs. [55,56]. The deformation parameter $\left|\beta_{2}\right|$ is enhanced from the def-GHFB results to the AMD results for both Mg and $\mathrm{Ne}$ isotopes. Particularly near and in the island of inversion, i.e., at $N=16,18,20$, the $\left|\beta_{2}\right|$ values are zero in the def-GHFB results, but they are largely enhanced in the AMD results. This enhancement is originated from the correlations induced by the collective rotational motion through the AMP. These collective-motion effects are particularly important near and in the island of inversion.

In Ref. [57], deformed Skyrme Hartree-Fock (def-SHF) calculations were done for $\mathrm{Mg}$ isotopes with two types of interactions $\mathrm{SkM}^{*}$ and SLy4, where the AMP was not employed. Around $N=20$, the densities are well deformed with $\mathrm{SkM}^{*}$, but not with SLy4. The resultant $\left|\beta_{2}\right|$ are then close to the AMD results when SkM* interaction is taken, in spite of the fact that the AMP is not performed in these def-SHF calculations.

Next we compare the neutron rms radius $\left\langle\boldsymbol{r}_{n}^{2}\right\rangle^{1 / 2}$ with the proton one $\left\langle\boldsymbol{r}_{p}^{2}\right\rangle^{1 / 2}$ in order to see the isovector component of matter density. Figure 8 shows the $A$ dependence of $\left\langle\boldsymbol{r}_{n}^{2}\right\rangle^{1 / 2}$ and $\left\langle\boldsymbol{r}_{p}^{2}\right\rangle^{1 / 2}$ for Mg isotopes. As expected, the neutron skin thickness

$$
\Delta R=\left\langle\boldsymbol{r}_{n}^{2}\right\rangle^{1 / 2}-\left\langle\boldsymbol{r}_{p}^{2}\right\rangle^{1 / 2}
$$

grows as $A$ increases with $Z$ fixed. In Fig. 9, the $\Delta R$ is plotted as a function of the asymmetric parameter $(N-Z) / A$ for $\mathrm{Mg}$ and $\mathrm{Ne}$ isotopes, where the AMD results for $\mathrm{Ne}$ isotopes are taken from Ref. [15]. The present results are consistent with the results of deformed Skyrme-HF (def-SHF) calculations with SLy4 interaction (dashed line) [64] rather than the relativistic mean-field calculations with NL3 interaction (dotted line) [65]. 


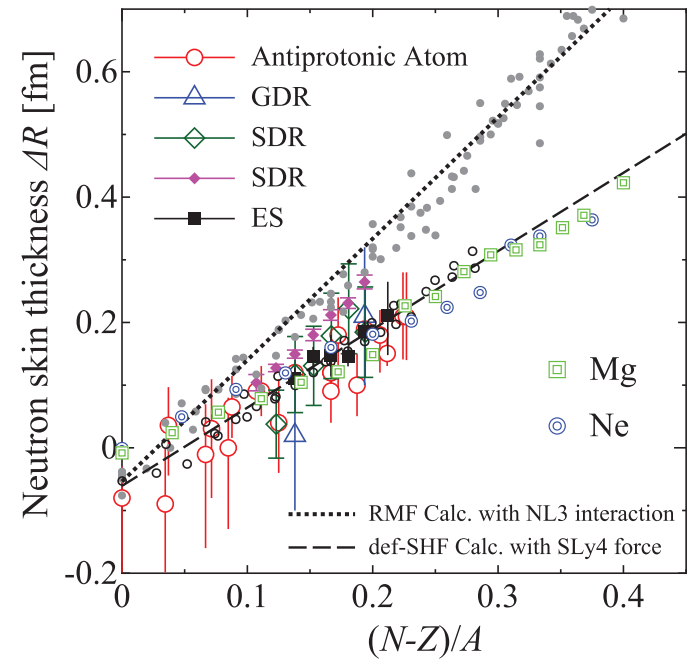

FIG. 9. (Color online) Neutron skin thickness $\Delta R$ in Eq. (26) as a function of asymmetric parameter $(N-Z) / A$. The double circles and double squares denote the AMD results for the $\mathrm{Mg}$ and $\mathrm{Ne}$ isotope, respectively. The case of ${ }^{31} \mathrm{Ne}$ is not plotted here, since the nucleus has a halo structure. Neutron skin thickness is also deduced from other measurements such as antiprotonic atoms (open circles) [58], giant dipole resonance (GDR) (open triangles) [59], spin dipole resonance (SDR) (open and closed diamonds) [60,61], and proton elastic scattering (ES) (closed squares) [62,63]. The AMD results are compared with the results of relativistic mean-field (RMF) calculations with NL3 interaction (small filled dots, whose average trend is represented by the dotted line) [65], and of deformed Skyrme-HF (def-SHF) calculations with SLy4 force (small open dots, whose average trend is represented by the dashed line) [64].

\section{DISCUSSIONS}

\section{A. Comparison between the AMD and def-WS models}

The results of the def-WS model are now compared with the results of fully microscopic AMD calculations in order to see the reliability of the phenomenological model. The def-WS model yields the same $I^{\pi}$ as AMD for ${ }^{24-40} \mathrm{Mg}$ except ${ }^{29} \mathrm{Mg}$. For ${ }^{29} \mathrm{Mg}$, the $I^{\pi}$ is $3 / 2^{+}$in AMD, which is consistent with the experimental data [52], but $1 / 2^{+}$in the def-WS model. This is because of the difference of the Coriolis coupling in the two models; its effect is larger in AMD than in def-WS. In ${ }^{29} \mathrm{Mg}$ the last odd-neutron occupies the [200 1/2] orbital, see Table IV, which has $\Omega=1 / 2$ ( $\Omega$ is the projection of the angular momentum on the symmetry axis). It is well known that the first-order Coriolis coupling changes the energy spectrum of the $\Omega=1 / 2$ rotational band [66]; if the effect is strong enough, the energies of the $I=3 / 2,7 / 2,11 / 2, \ldots$ sequence becomes lower than those of the $I=1 / 2,5 / 2,9 / 2, \ldots$ sequence. In the def-WS model the Coriolis coupling can be estimated by the so-called decoupling parameter; its calculated value is slightly larger than -1.0 in the present case, so that the inversion between the $I^{\pi}=3 / 2^{+}$and $I^{\pi}=1 / 2^{+}$energies does not occur. If the value is a bit smaller, the $I^{\pi}=3 / 2^{+}$state becomes the ground state also in the def-WS model.

The results of the AMD and def-WS models are compared for $\sigma_{\mathrm{R}}$ in Fig. 10. For ${ }^{24-36} \mathrm{Mg}$, the def-WS model (dashed line) well simulates the AMD results (solid line), but the former

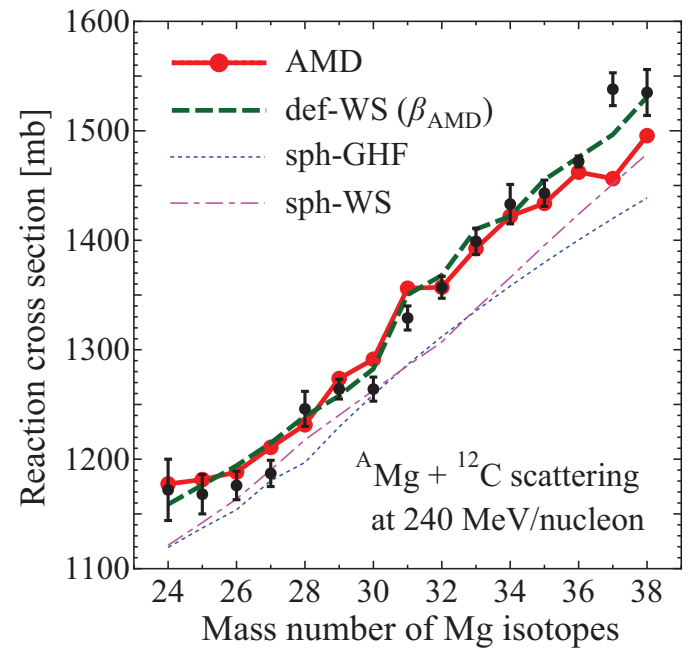

FIG. 10. (Color online) Comparison between AMD and def-WS calculations in reaction cross sections for $\mathrm{Mg}$ isotopes. The solid (dashed) line denotes the results of AMD (def-WS) calculations, whereas the thin dotted (dot-dashed) line stands for the results of sph-GHF (sph-WS) calculations. The experimental data are taken from Ref. [18].

overestimates the latter for ${ }^{37,38} \mathrm{Mg}$. To clarify the nature, we also plot the results of sph-WS and sph-GHF calculations that correspond to the spherical limit of def-WS and AMD calculations, respectively. The sph-WS results (thin dot-dashed line) are consistent with the sph-GHF results (thin dotted line) for ${ }^{24-36} \mathrm{Mg}$, but the former overshoots the latter for ${ }^{37,38} \mathrm{Mg}$. The parametrization of the sph-WS model is thus inappropriate for ${ }^{37,38} \mathrm{Mg}$ near the neutron drip line in comparison with the spherical HF result with Gogny-D1S force. Some correction should be made in the future; in particular, the depth of potential is assumed to be linear in the asymmetric parameter $(N-Z) / A$, see Eq. (A1), which may result in too shallow potentials compared to the sph-GHF for drip-line nuclei. It may be accidental that the def-WS model reproduces the measured $\sigma_{\mathrm{R}}$ for ${ }^{38} \mathrm{Mg}$. The fact that AMD calculations underestimate the measured $\sigma_{\mathrm{R}}$ for ${ }^{37,38} \mathrm{Mg}$ indicates that these are candidates for deformed halo nucleus.

\section{B. Results of AMD-RGM calculation for ${ }^{37} \mathrm{Mg}$}

As shown in Sec. III, the deduced matter radius of ${ }^{37} \mathrm{Mg}$ is quite large, and at the same time, the AMD calculation predicts large deformation $\left(\beta_{2} \sim 0.362\right)$ and very small separation energy $\left(S_{-1 n}=0.49 \mathrm{MeV}\right)$. These results suggest that ${ }^{37} \mathrm{Mg}$ is a candidate for deformed halo nucleus. On the other hand, the matter radius of ${ }^{37} \mathrm{Mg}$ calculated by AMD is much smaller than the one deduced from the measured $\sigma_{R}$. This might be because of the inaccuracy of the AMD density in its tail region. In this sense, we should solve the relative motion between the last neutron and the core $\left({ }^{36} \mathrm{Mg}\right)$ more precisely by using the following AMD-RGM framework [36], although the calculations are quite time consuming. This procedure is nothing but making a tail correction to AMD density.

In principle, the ground state $\Phi\left({ }^{37} \mathrm{Mg} ; I^{\pi}\right)$ of ${ }^{37} \mathrm{Mg}$ can be expanded in terms of the ground and excited states 


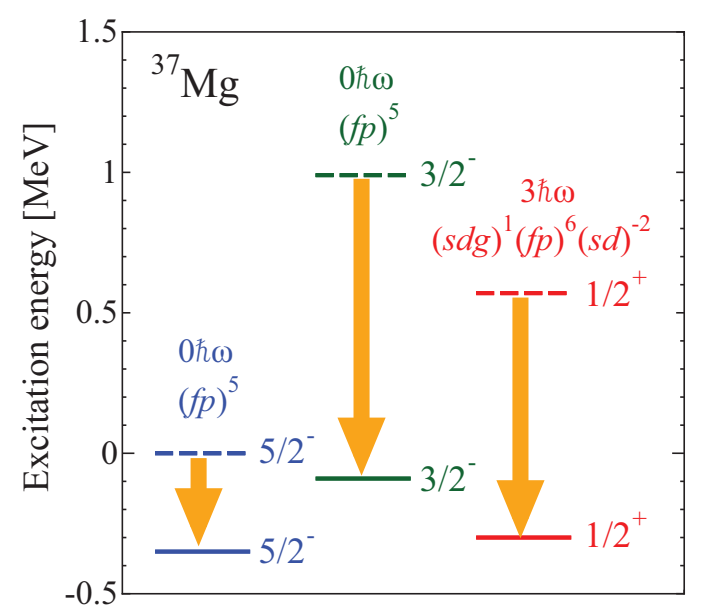

FIG. 11. (Color online) Energy spectrum of ${ }^{37} \mathrm{Mg}$. The dashed (solid) lines denote the results of AMD (AMD-RGM) calculations. All the excitation energies are plotted with reference to the energy of the $5 / 2^{-}$state calculated with AMD.

$\Phi\left({ }^{36} \mathrm{Mg} ; \tilde{I}_{i}^{\tilde{\tilde{\pi}}}\right)$ of ${ }^{36} \mathrm{Mg}$, where $\tilde{I}_{i}^{\tilde{\tilde{n}}}$ denotes the spin parity of ${ }^{36} \mathrm{Mg}$ in its $i$ th state. This means that the ground state of ${ }^{37} \mathrm{Mg}$ is described by the ${ }^{36} \mathrm{Mg}+n$ cluster model with core excitations. The cluster-model calculation can be done with the RGM in which the ground and excited states of ${ }^{36} \mathrm{Mg}$ are constructed by AMD:

$$
\Phi\left({ }^{37} \mathrm{Mg} ; I^{\pi}\right)=\sum_{i l j \tilde{I} \tilde{\pi}} \mathcal{A}\left\{R_{i l j}(r)\left[\left[Y_{l}(\hat{\boldsymbol{r}}) \chi_{n}\right]_{j} \Phi\left({ }^{36} \mathrm{Mg} ; \tilde{I}_{i}^{\tilde{\pi}}\right)\right]_{I^{\pi}}\right\},
$$

where $\chi_{n}$ is the spin wave function of last neutron and $R_{i l j}(r) Y_{l m}(\hat{\boldsymbol{r}})$ is the relative wave function between the last neutron and the core $\left({ }^{36} \mathrm{Mg}\right)$. All the excited states of ${ }^{36} \mathrm{Mg}$ below $8 \mathrm{MeV}$ obtained by the AMD calculation are included as $\Phi\left({ }^{36} \mathrm{Mg} ; \tilde{I}_{i}^{\tilde{\pi}}\right)$.

Figure 11 shows energy spectra of ${ }^{37} \mathrm{Mg}$ calculated with the AMD and AMD-RGM models. The deviation of the AMDRGM result (solid line) from the corresponding AMD result (dashed line) shows an energy gain due to the tail correction. Eventually, three bound states appear in the order of $I^{\pi}=$ $5 / 2^{-}, 1 / 2^{+}$, and $3 / 2^{-}$from the bottom. In AMD calculations, the main configuration of the $I^{\pi}=1 / 2^{+}$state for five valence neutrons corresponds to $(s d g)^{1}(f p)^{6}(s d)^{-2}$ in the spherical shell model, whereas the main configuration of the $I^{\pi}=5 / 2^{-}$ and $3 / 2^{-}$states is $(f p)^{5}$. The $I^{\pi}=1 / 2^{+}$state is quite exotic in the sense that one neutron is in the $0 g 1 d 2 s$ shells as a consequence of large deformation. These states are quite close in energy, and hence there is a possibility that the order is reversed in reality. We then assume that any one of the three states is the ground state.

In Fig. 12, the $\sigma_{\mathrm{R}}$ before and after the tail correction are shown for the three states. When the $5 / 2^{-}$state is the ground state, the calculated value of $\sigma_{\mathrm{R}}$ is little enhanced by the tail correction and still underestimates the measured $\sigma_{\mathrm{R}}$. This underestimation may not be resolved even if the $5 / 2^{-}$ state has smaller binding energy, since the last neutron is in

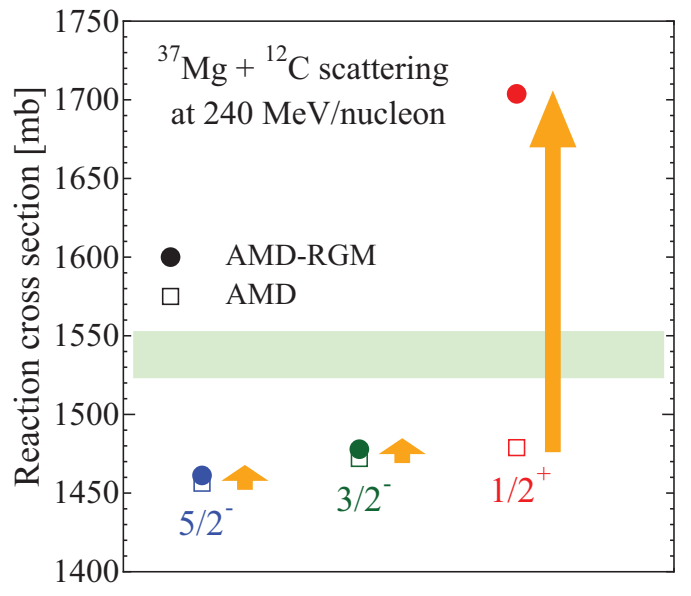

FIG. 12. (Color online) Reaction cross sections for ${ }^{37} \mathrm{Mg}$ calculated with the AMD and AMD-RGM methods. Open squares and closed circles stand for AMD and AMD-RGM results, respectively. It is assumed that any one of the $5 / 2^{-}, 1 / 2^{+}$, and $3 / 2^{-}$states is the ground state.

the [312 5/2] $\left(0 f_{7 / 2}\right.$-origin) orbital; see the single-particle energy for negative parity in Fig. 13(b). This was numerically confirmed in our previous work [18] by using the def-WS model, i.e., by changing the parameters of the def-WS potential slightly for the last neutron.

When the $1 / 2^{+}$state is the ground state, the last neutron occupies [440 1/2] $\left(0 g_{9 / 2}\right.$ origin) as shown in Fig. 13(a), where the $s$-wave halo can be formed. The calculated value of $\sigma_{\mathrm{R}}$ is largely enhanced by the tail correction and consequently overestimates the measured $\sigma_{\mathrm{R}}$. However the overestimation can be resolved if the $1 / 2^{+}$state has a larger binding energy by some effect. When the $3 / 2^{-}$state is the ground state, the calculated value of $\sigma_{\mathrm{R}}$ is little enhanced by the tail correction and still undershoots the measured $\sigma_{\mathrm{R}}$. In AMD calculations, the $3 / 2^{-}$state has the main component in which the last neutron is mainly coupled with not the ground state of ${ }^{36} \mathrm{Mg}$ but the excited $2^{+}$state, so that the last neutron is not weakly bound because of the core excitation. Consequently, the $\sigma_{\mathrm{R}}$ is hardly enhanced in AMD-RGM calculations. The $\sigma_{\mathrm{R}}$ may be enhanced, if the core excitation is suppressed by some effect.

The present AMD-RGM calculations thus cannot reproduce the measured $\sigma_{\mathrm{R}}$ perfectly. In nuclei near the drip line, in general, the kinetic energy is nearly canceled with the potential energy coming from the two-nucleon $(2 \mathrm{~N})$ central and spinorbit forces. This suggests that higher-order effects such as the $2 \mathrm{~N}$ tensor force and the three-nucleon $(3 \mathrm{~N})$ force become important. In our previous work [18] based on the def-WS model, the ground-state spin-parity is $I^{\pi}=5 / 2^{-}$, since the [312 5/2] $\left(0 f_{7 / 2}\right.$-origin $)$ orbital is slightly lower in energy than the others. To explain the measured large $\sigma_{\mathrm{R}}$ for ${ }^{37} \mathrm{Mg}$, we assumed that the last neutron is in the [321 1/2] $\left(1 p_{3 / 2}\right.$-origin) orbital. As an underlying mechanism of the inversion, we can consider the $2 \mathrm{~N}$ tensor force and the $3 \mathrm{~N}$ force, since it is reported that the $2 \mathrm{~N}$ tensor force reduces the energy difference between $0 f_{7 / 2}$ and $1 p_{3 / 2}$ levels in the spherical shell model [11] and the threebody force weakens the strength of the spin-orbit interaction in neutron-rich nuclei [67]. Further analyses along this line are 

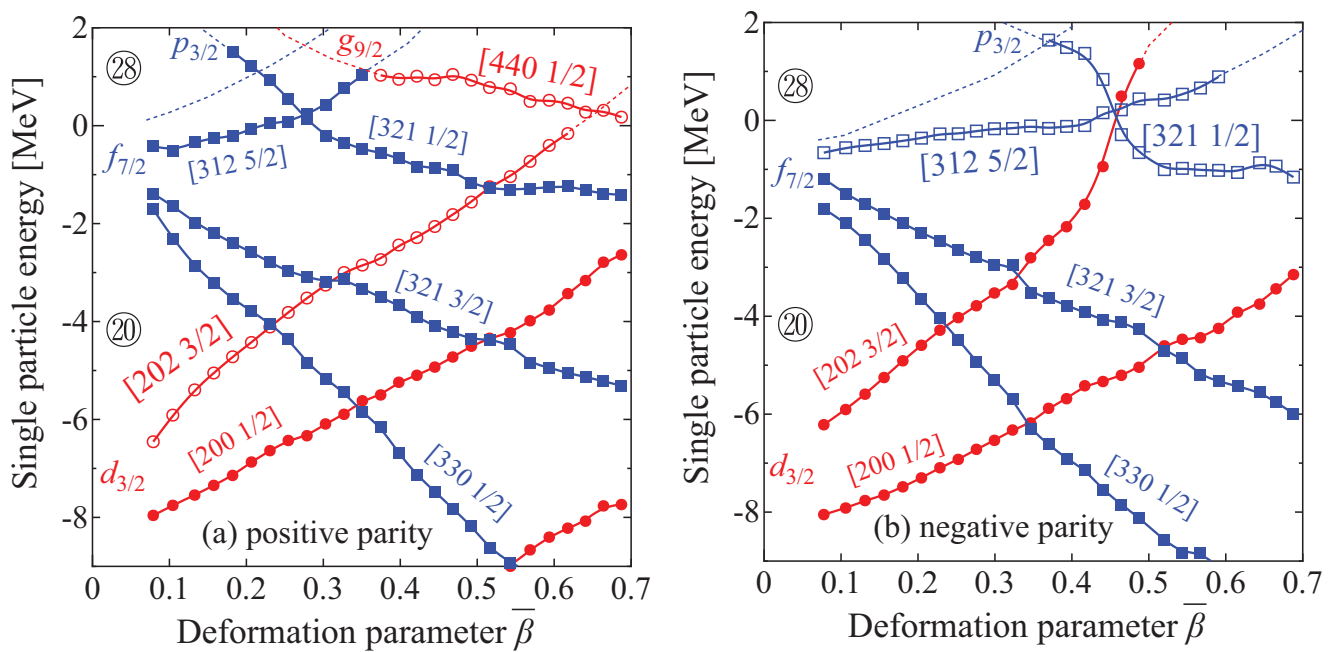

FIG. 13. (Color online) Neutron single-particle energies of ${ }^{37} \mathrm{Mg}$ for (a) positive parity and (b) negative parity. Filled (open) symbols mean the orbitals occupied by two (one) neutrons. Circles (squares) show the orbital where the amount of the positive-parity component is larger (smaller) than $50 \%$.

quite interesting. The analysis of ${ }^{38} \mathrm{Mg}$ is also an important future work after understanding the structure of ${ }^{37} \mathrm{Mg}$.

\section{SUMMARY}

We have determined matter radii of ${ }^{24-38} \mathrm{Mg}$ systematically from measured $\sigma_{\mathrm{R}}$, fine tuning the parameters of the def-WS model. This flexibility is an advantage of the def-WS model. The deduced matter radii are largely enhanced from the stable and spherical limit estimated by sph-GHF calculations with the Gogny-D1S interaction for stable spherical nuclei. Two-thirds of the enhancement come from nuclear deformation, whereas one-third is from neutron-skin and/or weak-binding effects.

Fully microscopic AMD calculations with the Gogny-D1S interaction, meanwhile, have no free parameter and hence high predictability, if the calculations are successful in reproducing existing experimental data systematically. The AMD calculations well reproduce measured ground-state properties (spin parity, total binding energy, and one-neutron separation energy) of $\mathrm{Mg}$ isotopes. The deduced matter radii can be also well reproduced for ${ }^{24-36} \mathrm{Mg}$. AMD is thus reliable and hence have high predictability. As for ${ }^{37,38} \mathrm{Mg}$, theoretical matter radii calculated with AMD are enhanced by deformation, but still considerably underestimate the deduced matter radii. This problem is not cured even with the more sophisticated AMDRGM framework. Further theoretical investigation should be done; e.g., the effective interaction Gogny-D1S force may not be best suited for the description of drip-line nuclei. This large enhancement of the measured matter radius suggests that ${ }^{37,38} \mathrm{Mg}$ are candidates for deformed halo nucleus.

Neutron-number $(N)$ dependence of deformation parameter $\beta_{2}$ is predicted by AMD. For both $\mathrm{Mg}$ and $\mathrm{Ne}$ isotopes, AMD calculations show an abrupt increase of $\beta_{2}$ at $N=19$, where the Nilsson orbitals originating from the spherical $0 f_{7 / 2}$ shell begin to be occupied. The starting point of the island of inversion is thus $N=19$. At $N=19 \sim 28$, the $\beta_{2}$ keep large values of around 0.4. Hence there seems to be no endpoint of the island of inversion. Moreover, the $N=20$ and 28 magicities disappear. Since ${ }^{40} \mathrm{Mg}$ with $N=28$ may be a drip-line nucleus, the island of inversion may not be an island, but a peninsula that reaches the neutron drip line. At $N=16,18$, and 20, deformation parameter $\beta_{2}$ vanishes in def-GHFB calculations with no AMP, but becomes large in AMD calculations with the AMP. The correlations induced by the collective rotational motion through the AMP are thus important.

Neutron-number dependence of neutron-skin thickness $(\Delta R)$ is also predicted by AMD. The AMD results for $\mathrm{Mg}$ and $\mathrm{Ne}$ isotopes are consistent with the results of SkyrmeHF calculations with SLy4 force rather than the relativistic mean-field calculations with NL3 interaction.

\section{ACKNOWLEDGMENTS}

The authors thank K. Ogata and H. Sakurai for fruitful discussions. This work is supported in part by Grant-in-Aid for Scientific Research (KAKENHI) from Japan Society for the Promotion of Science $(25.4319,24.4137,25.949$, 25400240, 22540285), and by Grant-in-Aid for Scientific Research on Innovative Areas from MEXT (2404: 24105008). The numerical calculations of this work were performed on the computing system in Research Institute for Information Technology of Kyushu University and the HITACHI SR16000 at KEK and YITP.

\section{APPENDIX A: WOODS-SAXON POTENTIAL PARAMETER SET}

In this paper, the strength $V_{0}$ of the WS potential in Eq. (22) is parameterized as

$$
V_{0}=-V \times\left(1 \pm \kappa \frac{N-Z}{A}\right), \quad\left\{\begin{array}{l}
+ \text { proton } \\
- \text { neutron }
\end{array}\right.
$$

with proton, neutron, and mass numbers, $Z, N$, and $A$. The WS potential is then characterized by the parameters, $V, \kappa, R_{0}, a$, and $\lambda$. We use the parameter set provided by Ramon Wyss [45]. 
TABLE III. The parameter set of the Woods-Saxon potential adopted in this work [45]. As other physical constants, $e^{2} /(\hbar c)=$ $137.03602, \hbar c=197.32891 \mathrm{MeV}$ fm, and $m c^{2}=938.9059 \mathrm{MeV}$ are taken.

\begin{tabular}{lcccccc}
\hline \hline$V(\mathrm{MeV})$ & $\kappa_{\mathrm{c}}$ & $\kappa_{\mathrm{so}}$ & $R_{0 \mathrm{c}}(\mathrm{fm})$ & $R_{0 \mathrm{so}}(\mathrm{fm})$ & $a(\mathrm{fm})$ & $\lambda_{\mathrm{so}}$ \\
\hline 53.7 & 0.63 & 0.25461 & $1.193 A^{1 / 3}+0.25$ & $0.969 \times R_{0 \mathrm{c}}$ & 0.68 & 26.847
\end{tabular}

The set is fitted to the moment of inertia and the quadrupole moment systematically for medium and heavy nuclei. The set has already used in some works with success [15,37]. The parameter set is shown in Table III; see Ref. [68] for the Coulomb part.

\section{APPENDIX B: RELATION OF DEFORMATION PARAMETERS BETWEEN AMD AND DEF-WS MODELS}

We show the relation between $\bar{\beta}$ in AMD and $\beta_{2}$ in the def-WS model. For simplicity, we only consider the axially symmetric deformation $(\gamma=0)$. Equation (24) in Sec. II is reduced to

$$
R(\theta)=R_{0} c_{v}\left(\beta_{2}\right)\left[1+\beta_{2} Y_{20}(\theta)\right],
$$

where $c_{v}$ is the factor assuring the volume conservation of nucleus. The relation between $\bar{\beta}$ and $\beta_{2}$ can be extracted analytically by considering the sharp-cut density

$$
\rho(\boldsymbol{r})=\rho_{0} \theta(R(\theta)-r)
$$

with $\rho_{0}=3 A /\left(4 \pi R_{0}^{3}\right)$. For this density, we analytically obtain

$$
\begin{aligned}
\left\langle x^{2}\right\rangle & =\int x^{2} \rho(\boldsymbol{r}) d \boldsymbol{r} \\
& =\frac{4 \rho_{0} R_{0}^{5} c_{v}^{5}}{15}\left(\pi-\frac{\sqrt{5 \pi}}{2} \beta_{2}+\frac{25}{14} \beta_{2}^{2}-\frac{5}{28} \sqrt{\frac{5}{\pi}} \beta_{2}^{3}+\cdots\right), \\
\left\langle y^{2}\right\rangle & =\left\langle x^{2}\right\rangle, \\
\left\langle z^{2}\right\rangle & \left.=\int z^{2} \rho(\boldsymbol{r}) d \boldsymbol{B} 3\right) \\
& =\frac{4 \rho_{0} R_{0}^{5} c_{v}^{5}}{15}\left(\pi+\sqrt{5 \pi} \beta_{2}+\frac{55}{14} \beta_{2}^{2}+\frac{10}{7} \sqrt{\frac{5}{\pi}} \beta_{2}^{3}+\cdots\right) .
\end{aligned}
$$

TABLE IV. The deformation parameter set $(\bar{\beta}, \bar{\gamma})$ in AMD and the corresponding standard set $\left(\beta_{2}, \gamma\right)$ in the def-WS model for $\mathrm{Mg}$ isotopes. The Nilsson asymptotic quantum numbers of last neutron are listed in the last column.

\begin{tabular}{llcccc}
\hline \hline nuclide & $\bar{\beta}$ & $\bar{\gamma}$ & $\beta_{2}$ & $\gamma$ & {$\left[N, n_{3}, \Lambda, \Omega\right]$ for last- $n$} \\
\hline${ }^{24} \mathrm{Mg}$ & 0.42 & $0^{\circ}$ & 0.434 & $0^{\circ}$ & {$[2113 / 2]$} \\
${ }^{25} \mathrm{Mg}$ & 0.40 & $0^{\circ}$ & 0.411 & $0^{\circ}$ & {$[2025 / 2]$} \\
${ }^{26} \mathrm{Mg}$ & 0.375 & $0^{\circ}$ & 0.384 & $0^{\circ}$ & {$[2025 / 2]$} \\
${ }^{27} \mathrm{Mg}$ & 0.35 & $0^{\circ}$ & 0.357 & $0^{\circ}$ & {$[2111 / 2]$} \\
${ }^{28} \mathrm{Mg}$ & 0.35 & $0^{\circ}$ & 0.357 & $0^{\circ}$ & {$[2111 / 2]$} \\
${ }^{29} \mathrm{Mg}$ & 0.295 & $0^{\circ}$ & 0.298 & $0^{\circ}$ & {$[2001 / 2]$} \\
${ }^{30} \mathrm{Mg}$ & 0.285 & 25 & $0.291^{\mathrm{a}}$ & $25.8^{\circ}$ & {$[2001 / 2]$} \\
${ }^{31} \mathrm{Mg}$ & 0.44 & $0^{\circ}$ & 0.456 & $0^{\circ}$ & {$[2001 / 2]$} \\
${ }^{32} \mathrm{Mg}$ & 0.395 & $0^{\circ}$ & 0.406 & $0^{\circ}$ & {$[2001 / 2]$} \\
${ }^{33} \mathrm{Mg}$ & 0.44 & $0^{\circ}$ & 0.456 & $0^{\circ}$ & {$[3213 / 2]$} \\
${ }^{34} \mathrm{Mg}$ & 0.35 & $0^{\circ}$ & 0.357 & $0^{\circ}$ & {$[3213 / 2]$} \\
${ }^{35} \mathrm{Mg}$ & 0.40 & $0^{\circ}$ & 0.411 & $0^{\circ}$ & {$[2023 / 2]$} \\
${ }^{36} \mathrm{Mg}$ & 0.39 & $0^{\circ}$ & 0.400 & $0^{\circ}$ & {$[2023 / 2]$} \\
${ }^{37} \mathrm{Mg}$ & 0.355 & $0^{\circ}$ & 0.362 & $0^{\circ}$ & {$[3125 / 2]$} \\
${ }^{38} \mathrm{Mg}$ & 0.38 & $0^{\circ}$ & 0.389 & $0^{\circ}$ & {$[3125 / 2]$} \\
\hline \hline
\end{tabular}

This value is obtained by assuming $\bar{\gamma}$ is finite. The value will become 0.288 if $\bar{\gamma}=0$; see Ref. [15] for details.

Combining these equations with Eq. (16) leads to the relation between $\bar{\beta}$ and $\beta_{2}$ as

$$
\begin{aligned}
\bar{\beta} & =\frac{1}{3} \sqrt{\frac{4 \pi}{5}} \ln \left(\frac{\left\langle z^{2}\right\rangle}{\left\langle x^{2}\right\rangle}\right) \\
& =\beta_{2}+\frac{1}{28} \sqrt{\frac{5}{\pi}} \beta_{2}^{2}-\frac{25}{28 \pi} \beta_{2}^{3}+\cdots
\end{aligned}
$$

The polynomial expression up to third order works well for $-0.6 \leqq \beta_{2} \leqq 0.6$. Obviously, we get $\bar{\beta}=\beta_{2}$ for small deformation.

Table IV lists the AMD deformation parameter set $(\bar{\beta}, \bar{\gamma})$ and the corresponding standard set $\left(\beta_{2}, \gamma\right)$ in the def-WS model for $\mathrm{Mg}$ isotopes. For ${ }^{24-38} \mathrm{Mg}$, only ${ }^{30} \mathrm{Mg}$ has nonzero $\bar{\gamma}$, but we simply set $\gamma$ to zero. This procedure is justified, since $\gamma$ deformation little affects $\sigma_{\mathrm{R}}[15]$.
[1] R. Klapisch et al., Phys. Rev. Lett. 23, 652 (1969).

[2] C. Thibault et al., Phys. Rev. C 12, 644 (1975).

[3] E. K. Warburton, J. A. Becker, and B. A. Brown, Phys. Rev. C 41, 1147 (1990).

[4] N. Orr et al., Phys. Lett. B 258, 29 (1991).

[5] T. Motobayashi et al., Phys. Lett. B 346, 9 (1995).

[6] E. Caurier, F. Nowacki, A. Poves, and J. Retamosa, Phys. Rev. C 58, 2033 (1998).

[7] Y. Utsuno, T. Otsuka, T. Mizusaki, and M. Honma, Phys. Rev. C 60, 054315 (1999).
[8] H. Iwasaki et al., Phys. Lett. B 522, 227 (2001).

[9] Y. Yanagisawa et al., Phys. Lett. B 566, 84 (2003).

[10] T. Otsuka, T. Suzuki, R. Fujimoto, H. Grawe, and Y. Akaishi, Phys. Rev. Lett. 95, 232502 (2005).

[11] T. Otsuka, T. Suzuki, M. Honma, Y. Utsuno, N. Tsunoda, K. Tsukiyama, and M. Hjorth-Jensen, Phys. Rev. Lett. 104, 012501 (2010).

[12] I. Tanihata et al., Phys. Lett. B 289, 261 (1992); I. Tanihata, J. Phys. G 22, 157 (1996).

[13] A. S. Jensen et al., Rev. Mod. Phys. 76, 215 (2004). 
[14] B. Jonson, Phys. Rep. 389, 1 (2004).

[15] T. Sumi, K. Minomo, S. Tagami, M. Kimura, T. Matsumoto, K. Ogata, Y. R. Shimizu, and M. Yahiro, Phys. Rev. C 85, 064613 (2012).

[16] T. Nakamura et al., Phys. Rev. Lett. 103, 262501 (2009).

[17] M. Takechi et al., Phys. Lett. B 707, 357 (2010).

[18] M. Takechi et al., EPJ Web of Conferences 66, 02101 (2014).

[19] G. Bertsch, J. Borysowicz, H. McManus, and W. G. Love, Nucl. Phys. A 284, 399 (1977).

[20] J.-P. Jeukenne, A. Lejeune, and C. Mahaux, Phys. Rev. C 16, 80 (1977); Phys. Rep. 25, 83 (1976).

[21] F. A. Brieva and J. R. Rook, Nucl. Phys. A 291, 299 (1977); 291, 317 (1977); 297, 206 (1978).

[22] G. R. Satchler and W. G. Love, Phys. Rep. 55, 183 (1979).

[23] G. R. Satchler, Direct Nuclear Reactions (Oxford University Press, Oxford, 1983).

[24] N. Yamaguchi, S. Nagata, and T. Matsuda, Prog. Theor. Phys. 70, 459 (1983); N. Yamaguchi, S. Nagata, and J. Michiyama, ibid. 76, 1289 (1986).

[25] L. Rikus, K. Nakano, and H. V. von Geramb, Nucl. Phys. A 414, 413 (1984); L. Rikus and H. V. von Geramb, ibid. 426, 496 (1984).

[26] K. Amos, P. J. Dortmans, H. V. von Geramb, S. Karataglidis, and J. Raynal, in Advanced Nuclear Physics, edited by J. W. Negele and E. Vogt (Plenum, New York, 2000), Vol. 25, p. 275.

[27] D. T. Khoa, W. von Oertzen, H. G. Bohlen, and S. Ohkubo, J. Phys. G 34, R111 (2007).

[28] T. Furumoto, Y. Sakuragi, and Y. Yamamoto, Phys. Rev. C 78, 044610 (2008); 79, 011601(R) (2009); 80, 044614 (2009).

[29] M. Toyokawa, K. Minomo, and M. Yahiro, Phys. Rev. C 88, 054602 (2013).

[30] M. Yahiro, K. Ogata, and K. Minomo, Prog. Theor. Phys. 126, 167 (2011).

[31] J. F. Berger, M. Girod, and D. Gogny, Comput. Phys. Commun. 63, 365 (1991).

[32] M. Kimura and H. Horiuchi, Prog. Theor. Phys. 111, 841 (2004).

[33] M. Kimura, Phys. Rev. C 75, 041302 (2007).

[34] R. Rodríguez-Guzmán, J. L. Egido, and L. M. Robledo, Nucl. Phys. A 709, 201 (2002).

[35] R. R. Rodríguez-Guzmán, J. L. Egido, and L. M. Robledo, Eur. Phys. J. A 17, 37 (2003).

[36] K. Minomo, T. Sumi, M. Kimura, K. Ogata, Y. R. Shimizu, and M. Yahiro, Phys. Rev. Lett. 108, 052503 (2012).

[37] K. Minomo, T. Sumi, M. Kimura, K. Ogata, Y. R. Shimizu, and M. Yahiro, Phys. Rev. C 84, 034602 (2011).
[38] K. M. Watson, Phys. Rev. 89, 575 (1953).

[39] A. K. Kerman, H. McManus, and R. M. Thaler, Ann. Phys. (NY) 8, 551 (1959).

[40] M. Yahiro, K. Minomo, K. Ogata, and M. Kawai, Prog. Theor. Phys. 120, 767 (2008).

[41] B. Sinha, Phys. Rep. 20, 1 (1975); B. Sinha and S. A. Moszkowski, Phys. Lett. B 81, 289 (1979).

[42] T. Furumoto, Y. Sakuragi, and Y. Yamamoto, Phys. Rev. C 82, 044612 (2010).

[43] K. Minomo, K. Ogata, M. Kohno, Y. R. Shimizu, and M. Yahiro, J. Phys. G 37, 085011 (2010).

[44] K. Hagino, T. Takehi, and N. Takigawa, Phys. Rev. C 74, 037601 (2006).

[45] R. Wyss (private communication).

[46] H. Nakada, Nucl. Phys. A 808, 47 (2008)

[47] H. de Vries, C. W. de Jager, and C. de Vries, At. Data Nucl. Data Tables 36, 495 (1987).

[48] M. Takechi et al., Phys. Rev. C 79, 061601(R) (2009).

[49] L. Chulkov et al., Nucl. Phys. A 603, 219 (1996).

[50] T. Suzuki et al., Phys. Rev. Lett. 75, 3241 (1995).

[51] S. Kox et al., Nucl. Phys. A 420, 162 (1984).

[52] G. Audi et al., Chin. Phys. C 36, 1157 (2012).

[53] T. Baumann et al., Nature (London) 449, 1022 (2007).

[54] P. Doornenbal et al., Phys. Rev. Lett. 111, 212502 (2013).

[55] S. Hilaire and M. Girod, Eur. Phys. J. A 33, 237 (2007).

[56] http://www-phynu.cea.fr/science_en_ligne/carte_potentiels _microscopiques/carte_potentiel_nucleaire_eng.htm.

[57] W. Horiuchi, T. Inakura, T. Nakatsukasa, and Y. Suzuki, Phys. Rev. C 86, 024614 (2012).

[58] A. Trzcinska, J. Jastrzebski, P. Lubinski, F. J. Hartmann, R. Schmidt, T. von Egidy, and B. Klos, Phys. Rev. Lett. 87, 082501 (2001).

[59] A. Krasznahorkay et al., Nucl. Phys. A 567, 521 (1994).

[60] A. Krasznahorkay et al., Phys. Rev. Lett. 82, 3216 (1999).

[61] A. Krasznahorkay et al., Nucl. Phys. A 731, 224 (2004).

[62] S. Terashima et al., Phys. Rev. C 77, 024317 (2008).

[63] J. Zenihiro et al., Phys. Rev. C 82, 044611 (2010).

[64] P. Sarriguren, M. K. Gaidarov, E. M. de Guerra, and A. N. Antonov, Phys. Rev. C 76, 044322 (2007).

[65] G. A. Lalazissis, D. Vretenar, and P. Ring, Phys. Rev. C 57, 2294 (1998).

[66] A. Bohr and B. R. Mottelson, Nuclear Structure (Benjamin, New York, 1975), Vol. II.

[67] M. Kohno, Phys. Rev. C 86, 061301 (2012).

[68] T. Shoji and Y. R. Shimizu, Prog. Theor. Phys. 121, 319 (2009). 